\title{
SPECTRO-ANGUlar OPTICAl BIOSENSOR Based On Surface Plasmon Resonance OPERATING In THE VISIBLE SPECTRUM
}

\section{Sandrine Filion Côté}

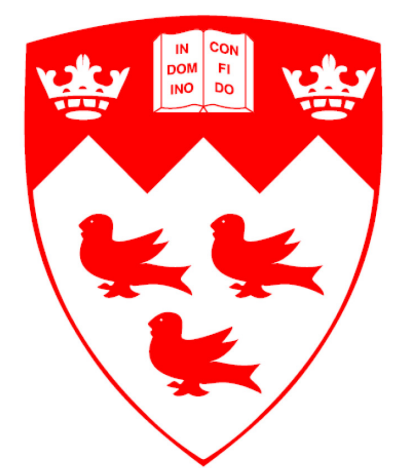

Department of Electrical and Computer Engineering

McGill University, Montréal August 2011

A thesis submitted to McGill University in partial fulfillment of the requirements of the degree of Master's of Engineering

(C) 2011 Sandrine Filion Côté 


\section{Abstract}

Surface plasmon resonance (SPR) is one of the most widely used methods to implement biosensors because of its label-free and sensitive detection. Surface plasmon resonance allows the change in the refractive index of a sample to be measured accurately by the analysis of the light reflecting at a metal-dielectric interface. A way to increase the sensitivity of SPR biosensors was found in fabricating a spectro-angular SPR biosensor and using of a newly developed data processing method called the Double Projection Method. The objective of the work presented in this thesis is to improve further the detection limit of the spectro-angular biosensor by upgrading the cameras used for the data acquisition. Simulations have shown that the spatial resolution and the data precision have a significant impact on the accuracy of the refractive index change measurement. In this thesis, simulation results are presented to justify the modifications of the experimental system and to estimate the expected improvement in the detection limit of the spectro-angular biosensor by the use of higher spatial resolution and higher data precision cameras. The new design as well as the components purchased for the experimental set-up are detailed. 


\section{Sommaire}

La résonance plasmonique de surface (SPR) est une méthode très utilisée pour la réalisation de biocapteurs grâce à sa détection très sensible et sans marqueur. La résonance plasmonique de surface permet une mesure précise du changement de l'indice de réfraction d'un échantillon par l'analyse de la lumière reflétant sur l'interface entre un métal et un diélectrique. Il a été démontré que la sensibilité des biocapteurs SPR puisse être accrue par la fabrication d'un biocapteur SPR spectroangulaire utilisant une nouvelle méthode de traitement des données appelée Double Projection Method (la méthode par double projection). L'objectif du travail présenté dans cette thèse est d'améliorer la limite de détection du biocapteur spectro-angulaire grâce à l'utilisation de caméras plus performantes pour la cueillette de données. Des simulations ont démontré que la résolution spatiale et la précision des données ont un impact significatif sur la précision de la mesure du changement dans l'indice de réfraction. Dans cette thèse, des résultats de simulations seront présentés afin de justifier les modifications à apporter au système expérimental et de démontrer l'amélioration prévue de la limite de détection du biocapteur spectro-angulaire par l'utilisation de caméras plus performantes. La nouvelle conception ainsi que les composantes choisis pour le système expérimental seront détaillées. 


\section{Acknowledgements / Remerciements}

I would first like to thank my supervisor Professor Andrew Kirk for introducing me to the field of Photonics and particularly to allow me to work on a multidisciplinary project which met my interest for biomedical applications. His guidance during my Master's project was greatly appreciated. I would also like to thank Dr. Philip Roche for his constant support and the various discussions we had on both technical and general topics. I would like to thank as well Dr. Colin Alleyne who worked on my current project before me for his $\mathrm{PhD}$ thesis. It was nice that we stayed in touch and that we could discuss the project together, it helped me a lot. I also want to recognize the work of undergraduate students who added important features to the project: Songzhe Wang for his work on the wavelet analysis and Xue Hao Ying for the data acquisition program. Furthermore, I would like to thank Don Pavlasek for his amazing job in designing and fabricating the prism holder. Additionally, I would like to thank my colleagues of the Photonic Systems Group for their support and generous help. A special thanks to Chams Baker for answering all my questions. I would also like to acknowledge the financial support of the Natural Sciences and Engineering Research Council of Canada (NSERC) as well as Le Fonds Québécois de la Recherche sur la Nature et les Technologies (FQRNT).

De plus, je voudrais remercier de tout cœur ma famille pour leurs encouragements constants au cours de ma maîtrise. J'ai grandement apprécié leur support et leur aide et je les remercie de m'avoir permis de poursuivre mes études aux cycles supérieurs. Un merci particulier à Mark pour m'avoir également aidé avec la source de courant. Je voudrais aussi remercier chaleureusement mes amis pour les encouragements, les discussions et les fous rires. Merci! 


\section{Table of Contents}

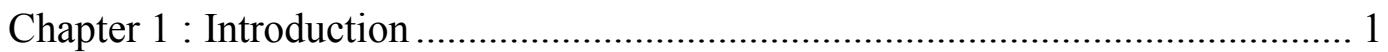

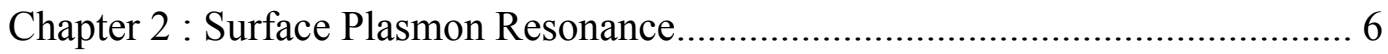

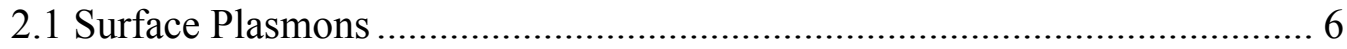

2.2 Surface Plasmon Coupling...................................................................... 8

2.3 Surface Plasmon Resonance ………………………………………….... 12

2.4 Interrogation Methods for Prism-Based Coupling........................................ 14

2.5 Refractive Index Change Measurement With SPR...................................... 17

2.6 Short-Range SPR, Long-Range SPR and Localized SPR ......................... 19

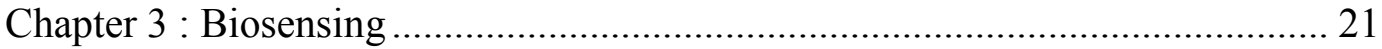

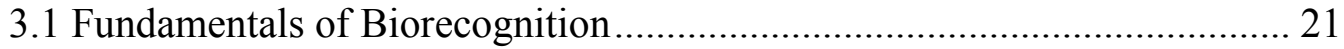

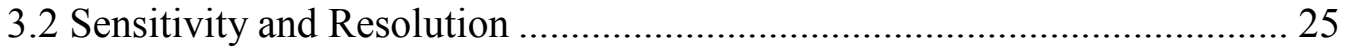

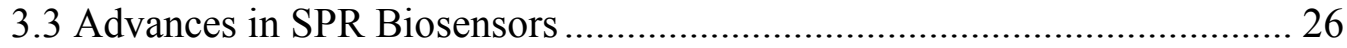

3.3.1 Prism-based Coupling ....................................................................... 27

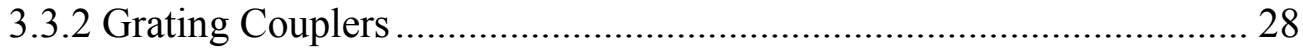

3.3.3 Waveguide Couplers ....................................................................... 28

Chapter 4 : General Concept of Spectro-Angular SPR Biosensing ....................... 30

4.1 System's Description........................................................................... 30

4.2 The Double Projection Method................................................................. 33

4.3 Noise Reduction: Temperature Control, Reference Channel and

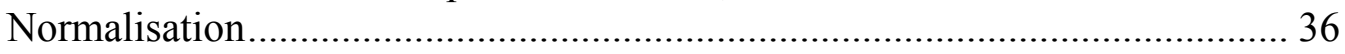

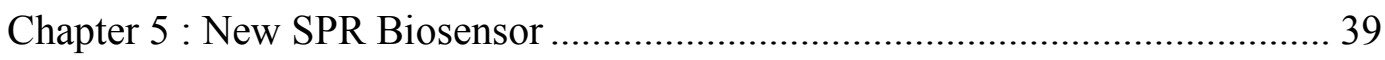

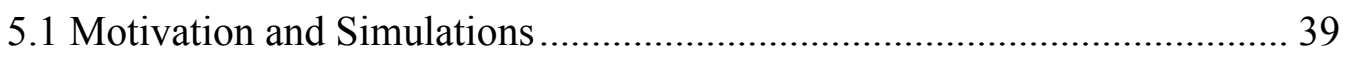

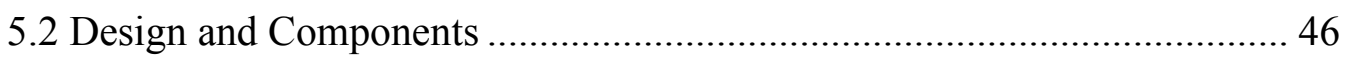

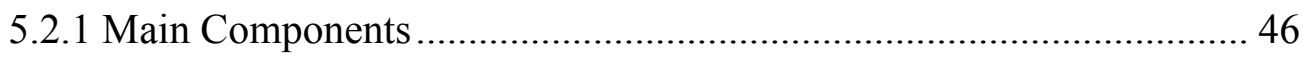

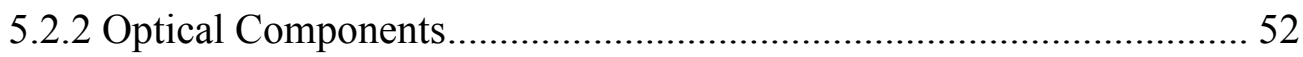

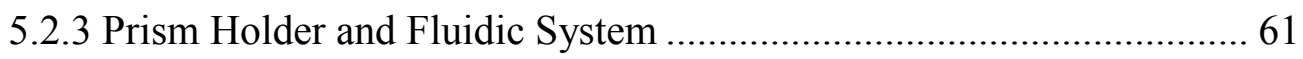

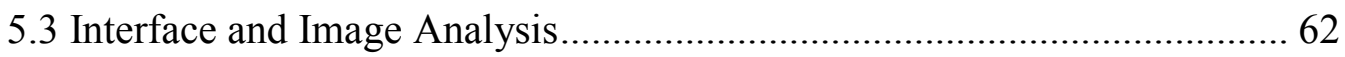

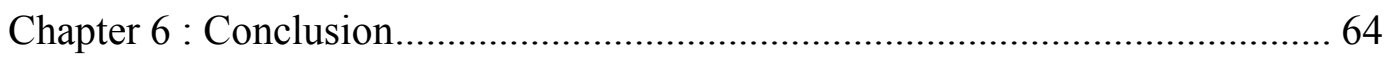

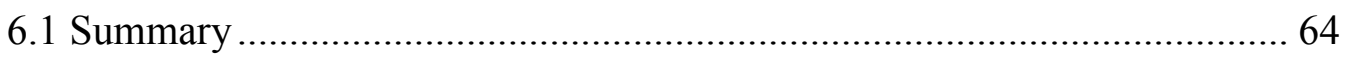

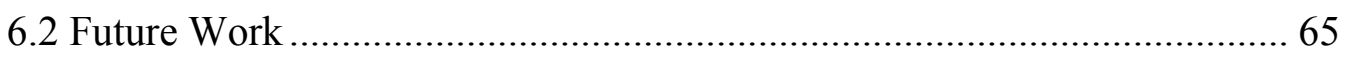

6.2.1 Fabrication and Testing of the Experimental Set-Up............................ 65 
6.2.2 One Camera For Both SPR Channels ............................................. 65

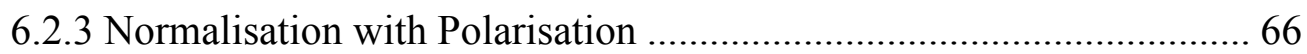

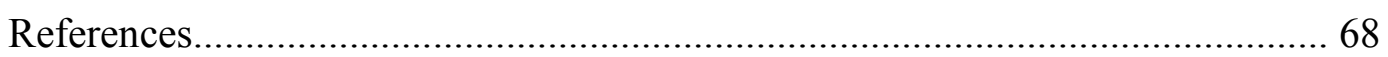




\section{List of Figures}

Figure 2.1 - Propagating surface plasmon at the interface of a metal and a dielectric (the direction of propagation is from left to right)

Figure 2.2 - Propagation constant, $\mathrm{k}_{\mathrm{x}}$, of the photon $\left(\mathrm{k}_{\mathrm{ph}}\right)$ and the surface plasmon $\left(\mathrm{k}_{\mathrm{sp}}\right)$ in water $(\mathrm{n}=1.33)$ and glass $(\mathrm{SF} 11-\mathrm{n}=1.78)$ using a $50 \mathrm{~nm}$ gold metal layer (parameters from [16]) and an incident angle of $54^{\circ}$

Figure 2.3 - ATR coupling methods: (a) Kretschmann configuration, (b) Otto configuration

Figure 2.4 - Grating coupling....

Figure 2.5 - Waveguide coupling.....

Figure 2.6 - Excitation of surface plasmon by TM and TE polarised light

Figure 2.7 - Reflected light with respect to angle of incidence at a wavelength of $630 \mathrm{~nm}$ with a dielectric refractive index of 1.333 refractive index unit (RIU)... 13 Figure 2.8 - Five different methods of interrogation: (a) angular, (b) wavelength, (c) intensity (d) interferometric and (e) spectro-angular.

Figure 2.9 - Phase shift with respect to refractive index for different angle of incidence [22]. The dynamic range in this case is 0.002 RIU.

Figure 2.10 - Shift in the resonance conditions (incident angle) reflecting the change in refractive index of the sample at a wavelength of $630 \mathrm{~nm}$.

Figure 2.11 - Localized surface plasmon 20

Figure 3.1 - Decaying fields in gold $(50 \mathrm{~nm})$ and water $(\mathrm{n}=1.33)$ for $630 \mathrm{~nm}$ and $850 \mathrm{~nm}$.

Figure 3.2 - Sensing surface with BRE (left) and binding of analyte to the BRE (right).

Figure 4.1 - Design of the spectro-angular biosensor [41] 32

Figure 4.2 - Spectro-angular SPR response for a refractive index of 1.33 RIU ... 32

Figure 4.3 - Spectro-angular SPR response for a refractive index of 1.4 RIU .... 33

Figure 4.4 - Summary of the Double Projection Method for refractive index estimation [14] 
Figure 5.1 - RMS Error for the estimation of a refractive index change of $10^{-6}$ and a training set with intervals of 0.002 RIU for different camera resolutions (256x256, 512x512 and 1024x 1024) and for different ADC levels (8-bit and 16bit). The noise added for each pixel (x-axis) is Gaussian with a standard deviation of $2^{\mathrm{Nb}-1} / 2^{\mathrm{n}}-1$ where $\mathrm{N}_{\mathrm{b}}$ is the number of bit of noise and $\mathrm{n}$ is the number of bit used in the ADC.

Figure 5.2 - Spectro-angular SPR response centered at $850 \mathrm{~nm}$ for a refractive index of 1.33 RIU

Figure 5.3 - Spectro-angular SPR response centered at $630 \mathrm{~nm}$ for a refractive index of 1.33 RIU

Figure 5.4 - SPR dip at three different wavelengths across the visible spectrum (500 nm, $600 \mathrm{~nm}$ and $700 \mathrm{~nm}$ ). The refractive index of the dielectric is $1.33 \mathrm{RIU}$.

Figure 5.5 - Output spectrum of the fibre-coupled LED measured by an optical

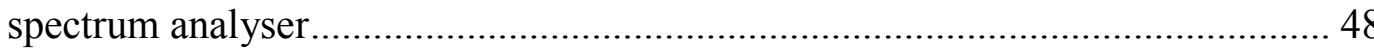

Figure 5.6 - Ray tracing for an extended source (multimode optical fibre)......... 52 Figure 5.7 - Effect of the collimation of an extended source on the angular resolution at the image plane. $\mathrm{L}_{1}$ is a spherical collimating lens and $\mathrm{L}_{2}$ and $\mathrm{L}_{3}$ are identical spherical focusing and collimating lenses........................................... 53

Figure 5.8 - A 1024 x 1024 ideal SPR image ................................................ 55

Figure 5.9 - A 1024 x 1024 blurred SPR image ............................................... 55

Figure 5.10 - Rays bending due to the change in the surrounding refractive index

Figure 5.11 - Rays entering the triangular prism with an angle $\alpha$ and reflecting on the gold with an angle $\theta$ 58

Figure 5.12 - Diffraction of an incident beam on a grating and focusing onto an image plane with a lens. 59

Figure 5.13 - Prism holder and fluidic system 62 


\section{List of Tables}

Table 2.1 - Theoretical sensitivity and FWHM of the SPR curve for $630 \mathrm{~nm}$ and $850 \mathrm{~nm}$ for angular SPR measurement. The metals have a thickness of $50 \mathrm{~nm}$ and the refractive index of the dielectric is around 1.33. The values for gold and silver are taken from [16] and the values for SF11 and BK7 are from [19]. 19

Table 3.1 - Theoretical propagation length, $\mathrm{L}$, and sensing region thickness, $\mathrm{z}_{\mathrm{d}}$, for $630 \mathrm{~nm}$ and $850 \mathrm{~nm}$. The metals, gold and silver, have a thickness of $50 \mathrm{~nm}$ and the refractive index of the dielectric is 1.33 . The values for gold and silver are taken from [16]. 22

Table 3.2 - Summary of the limit of detection for the different SPR methods..... 29 Table 5.1 - RMS estimation error for different refractive index changes using the same cameras 45

Table 5.2 - RMS estimation error for different refractive index changes using different cameras. 46

Table 5.3 - Specifications of the fibre-coupled LED from Doric lenses .... 48

Table 5.4 - Comparison between the specifications of the Micronviewer and the Pike cameras

Table 5.5 - Parameters of the AVT Pike camera and Kodak Sensor ...................... 50

Table 5.6 - Performance of the Kodak sensor.

Table 5.7 - Theoretical RMS estimation error for blurred 1024x1024 SPR images at a center wavelength of $630 \mathrm{~nm}$. 14-bit of quantization noise is considered in both the noise-free and noisy cases. 


\section{Abbreviations}

$\begin{array}{ll}\text { ADC } & \text { Analog-to-digital converter } \\ \text { ATR } & \text { Attenuated total reflection } \\ \text { BRE } & \text { Biological recognition element } \\ \text { CCD } & \text { Charge-coupled device } \\ \text { DPM } & \text { Double projection method } \\ \text { FWHM } & \text { Full width at half maximum } \\ \text { LSPR } & \text { Localized surface plasmon resonance } \\ \text { NP } & \text { Nanoparticle } \\ \text { RI } & \text { Refractive index } \\ \text { RIU } & \text { Refractive index unit } \\ \text { RMS } & \text { Root-mean-square } \\ \text { SLD } & \text { Super luminescent diode } \\ \text { SNR } & \text { Signal-to-noise ratio } \\ \text { SP } & \text { Surface plasmon } \\ \text { SPR } & \text { Surface plasmon resonance } \\ \text { SVD } & \text { Singular value decomposition } \\ \text { TE } & \text { Transverse electric } \\ \text { TEC } & \text { Thermo-electric cooler } \\ \text { TM } & \text { Transverse magnetic }\end{array}$




\section{Chapter 1: InTRODUCTION}

Sensors are useful in many aspects of our life. From the thermometer, passing by the accelerometers in cars to sensors for blood glucose monitoring used by diabetics, they ease our life and give vital information on ourselves and our environment. Sensors are devices used to detect changes in the state of a sample. In the examples given before, the change in state could be the rise in temperature in air or water, the decrease in acceleration of a car or the level of sugar in someone's blood. Among sensors, biosensors are very much valued as they are detecting and measuring biomolecules that can't be measured by eye and that means so much. They are the instruments for diagnosis, either to help detect cancer or to declare water or food safe for consumption, etc. Biosensors are widely used in medical and environmental applications; they are used for food quality and safety, medical diagnostics and environmental monitoring. These devices detect and measure low quantities of biomolecules such as biochemical contaminants, vitamins, proteins, drugs, etc.

There are two important parts in biosensors: the biorecognition and the transduction. The biorecognition is the specific detection of the biomolecules of interest while the transduction is a mean used to translate the biological event into a readable signal, usually an electronic signal, to be analysed. The biorecognition is done either by immobilizing the biomolecules on the sensing surface with antibodies or by labelling the biomolecules. Labelling is done by attaching molecules, nanoparticles, fluorophores, etc to the biomolecules of interest to signal their quantity in the sample.

The transduction can be implemented by multiple ways, mechanically, electrically or optically. In the first category, a difference in mass due to the binding of biomolecules will produce a readable signal. For example, biosensors are fabricated using microcantilevers $[1,2]$ where a piece of polymer bends due to the 
pressure of biomolecules attached to the surface. The mechanical stress in the micorcantilever is measured using a piezoresistor and the detection is possible with the analysis of the electrical output. Another option is to use quartz crystal microbalance biosensors. An electrical signal will go through a quartz crystal resonator and the resonator will vibrate at the resonant frequency. The frequency of oscillation depends on the mass of the biomolecules on the surface and is measured with a quartz crystal microbalance [3].

For electrical biosensors, the transduction is done by measuring the change in the electrical conductivity of a metal, polymer, liquid or else caused by the presence of biomolecules. In some biosensors, bacteria are used to detect the presence of toxic chemicals in a solution. For example, sulfur-oxidizing bacteria oxidize sulfur in presence of oxygen and produce sulfuric acid which changes the $\mathrm{pH}$ and the electrical conductivity of water [4]. In the presence of toxic chemicals, the bacteria stop their activity and therefore this can be used to monitor the toxicity. Another way to fabricate biosensors is to use carbon nanotubes. Single-walled carbon nanotubes were demonstrated as useful sensors since their conductivity changes with the attachment of biomolecules to their side. In particular a singlewalled carbon nanotube was shown to be an efficient gas sensor when it was coated with single-stranded DNA which served as the chemical recognition site $[5]$.

Biosensors can also be implemented optically. In this case, the change in the refractive index is measured as it can be related to a concentration of biomolecules. The change in refractive index due to the binding of biomolecules is very important since it is the refractive index that dictates the propagation properties of light. This is useful when waveguides are considered for biosensing. The properties of the mode propagating in the waveguide core depend on the refractive indices of the core and the cladding. Therefore, the presence of biomolecules in the cladding produces a change in the output reading of the waveguide. Waveguide biosensors are compact and have great potential to be 
used as point of care systems [6]. Additionally, a Mach-Zehnder interferometer can be turned into a sensor. Often used as an electro-optic modulation device for optical fibre communications, a Mach-Zehnder interferometer measures small differences in phase between two beams. The phase difference is either due to the difference in path length or in the difference in the refractive indices of both paths. Therefore, Mach-Zehnder interferometers can be used as sensing devices where one path is the reference and the other is the signal with a change in refractive index due to the adsorption of biomolecules [7]. A popular method for implementing biosensors is fluorescence [8]. Biomolecules of interest are tagged with fluorescent nanoparticles called fluorophores. Fluorophores absorb light energy at a specific wavelength and emit light at a longer wavelength. This way by illuminating the sample with the specific absorbing wavelength of the fluorophores and measuring the emitted light can give information on the concentration of biomolecules. The most widely used method for detection of food allergens in the food industry is the enzyme-linked immunosorbent assays (ELISA) that involves tagging the biomolecules of interest with enzymes that produce a visible signal. ELISA is mainly used for its ease of use and its fast and sensitive detection. ELISA is a standard biosensing test and commercial test kits are available $[9,10]$.

Finally, surface plasmon resonance (SPR) is one of the most widely used methods to implement biosensors [11]. Since the first demonstration of SPR biosensing in $1982[12,13]$, this method has become increasingly popular for its label-free and sensitive detection. Surface plasmon resonance allows the change in the refractive index of a sample to be measured accurately by the analysis of the light reflecting on a metal-dielectric interface. The main advantage of surface plasmon resonance biosensors is that they allow label-free detection. 'Labelling' a molecules involves binding a label to it which is often an antibody attached to a luminescent particle. The process of labelling adds additional steps to the detection process and therefore increases its complexity as well as adds cost due to the additional use of reagents. However, labelling is still a widely used method and there are specific 
measurements, such as single cell detection, where the labelling is required. Unless the labelling is necessary, label-free detection is preferred. .

Besides label-free detection, the real-time ability of the SPR method is very useful. Results can be obtained rapidly with SPR sensors and depending on the implementation method chosen it allows the sample to be monitored in real-time. This is interesting as the biosensor not only gives the refractive index difference between two states but also gives timing information. SPR biosensors can thus be used to study the binding kinetics of a receptor and ligand and find important parameters in chemistry, the association and dissociation constants. Another benefit of SPR sensing is the high throughput. SPR biosensors can be designed with multiple sensing channels to be interrogated simultaneously. In many applications, such as cancer detection, it is important to measure the concentration of multiple different biomolecules for a diagnosis to be possible and the high throughput ability of SPR biosensors allows results to be obtained faster.

Furthermore, SPR biosensors can be integrated and therefore be portable. The integration of biosensors is an attracting option as small devices are much more convenient since the medical practitioners could use them in their office with their patients or the patients could even have one at home for their personal use instead of waiting for a laboratory test. Additionally, research is conducted on the choice of material for the biosensors as well to reduce their cost in order to make them available to more people and decrease the cost of a test. The biosensor presented in this thesis performs highly sensitive refractive index estimation in real-time. The goal of the project is to improve the resolution of the device and does not include an increase in the throughput or the integration of the system.

A spectro-angular SPR biosensor was previously designed and fabricated and the results were obtained using a newly developed data processing method [14,15]. The output of a spectro-angular SPR biosensor is a two dimensional image representing the reflectivity of light with respect to both the angle of incidence 
and the wavelength. In order to process those images, an analysis method called the Double Projection Method was developed where the experimental images are compared with simulated images to extract the refractive index difference. In this current work, the goal is to improve the performance of the sensor by increasing the quality of the data analysed. As it was shown in Alleyne et al. [14], the spatial resolution and the data precision in the cameras make an important difference in terms of the performance of the biosensor. Therefore, the system could still be improved to achieve even greater performance by using higher resolution cameras. Charge-coupled device (CCD) cameras operating in the visible spectrum are small and offer very high resolution while maintaining a high data precision compared to cameras operating in the near infrared. The goal of this project is to redesign the SPR biosensor to function in the visible spectrum with newly purchased high resolution cameras in order to improve the sensitivity and obtain a lower detection limit approaching the theoretical limit of the sensor. It will be shown theoretically that the new camera parameters along with the change of wavelength to the visible spectrum improve the detection limit of the system by an order of magnitude.

In Chapter 2, a literature review of surface plasmon resonance will be presented. The different methods for coupling surface plasmons and how SPR can be used for detection will be described. Additionally, the different interrogation methods will be introduced. In Chapter 3, biosensing using surface plasmon resonance will be depicted and the performance of SPR biosensors will be put forward. Then the model of the biosensor which is the subject of this thesis will be outlined in Chapter 4 along with the data processing method. In Chapter 5, the new design of the system is detailed. Simulation results and the components chosen are shown along with the description of the data acquisition method. Finally, the future work on the biosensor is outlined in Chapter 6 with an emphasis on the usefulness and the possibility of using one camera for both SPR channels. 


\section{Chapter 2: Surface Plasmon Resonance}

\subsection{Surface Plasmons}

Surface plasmons (SP) are charge-density oscillations at the interface of two media that propagate parallel to the interface (Figure 2.1). These transversemagnetic (TM) polarised electromagnetic waves have their maxima on the interface and their intensity decays exponentially in both adjacent media. The dielectric constants of these media must be of opposite sign to allow the existence of the surface plasmons. Since metals have negative dielectric constants, they are often used to support surface plasmons. For biosensing purposes, the other dielectric is usually a liquid such as water and is the sample being interrogated. To excite surface plasmons, light must be incident on the thin metal film adjacent to the semi-infinite dielectric medium. The condition for excitation is that the momentum of the incident photons matches the momentum of the surface plasmon; in these conditions, resonance is reached and the energy of the photons is transferred to the plasmon. The propagation constant of the surface plasmon, $\mathrm{k}_{\mathrm{sp}}$, is given by equation 2.1 .

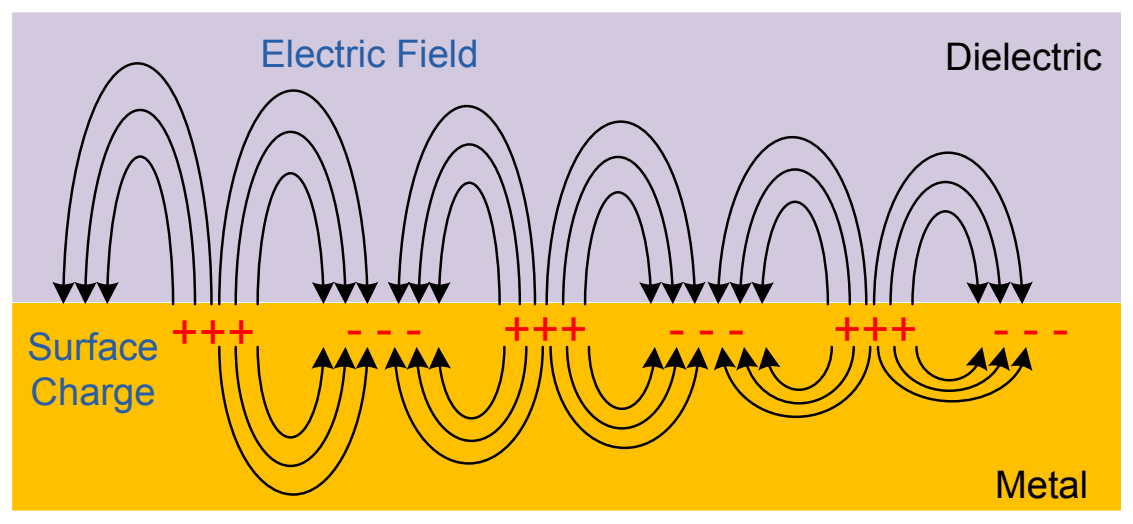

Figure 2.1 - Propagating surface plasmon at the interface of a metal and a dielectric (the direction of propagation is from left to right) 


$$
k_{s p}=\frac{2 \pi}{\lambda} \sqrt{\frac{\varepsilon_{m} \varepsilon_{d}}{\varepsilon_{m}+\varepsilon_{d}}}
$$

$\varepsilon_{\mathrm{m}}$, the dielectric constant of the metal, is a complex number and so is $\mathrm{k}_{\mathrm{sp}} . \varepsilon_{\mathrm{m}}$ is also highly dependent on the wavelength. $\varepsilon_{d}$ is the dielectric constant of the dielectric medium, usually real, and $\lambda$ is the wavelength of the incident light in vacuum. The propagation constant can be divided in real and imaginary parts. The real part, $\mathrm{k}_{\mathrm{sp}}$, determines the resonance conditions for coupling energy to the surface plasmons while the imaginary part, $\mathrm{k}_{\mathrm{sp}}$ ", is related to the propagation loss.

$$
k_{s p}=k_{s p}^{\prime}+j k_{s p}^{\prime \prime}=\frac{2 \pi}{\lambda} \sqrt{\frac{\varepsilon_{m}^{\prime} \varepsilon_{d}}{\varepsilon_{m}^{\prime}+\varepsilon_{d}}}+j \frac{2 \pi}{\lambda}\left(\frac{\varepsilon_{m}^{\prime} \varepsilon_{d}}{\varepsilon_{m}^{\prime}+\varepsilon_{d}}\right)^{3 / 2} \frac{\varepsilon_{m}^{\prime \prime}}{2 \varepsilon_{m}^{\prime 2}}
$$

$\varepsilon_{\mathrm{m}}{ }^{\prime}$ and $\varepsilon_{\mathrm{m}}{ }^{\prime \prime}$ are the real and imaginary parts of the dielectric constant of the metal respectively. For the photons to transfer energy to the surface plasmons, the propagation constant of the photons must be higher than that of the surface plasmon. The propagation constant of the photons, $\mathrm{k}_{\mathrm{ph}}$, is:

$$
k_{p h}=\frac{2 \pi}{\lambda} \sqrt{\varepsilon_{i}}=\frac{2 \pi}{\lambda} n_{i}
$$

$\varepsilon_{\mathrm{i}}$ and $\mathrm{n}_{\mathrm{i}}$ are the dielectric constant and the refractive index (related through $n=\sqrt{\varepsilon}$ ) of the medium in which the photons are propagating prior to reflect on the metal.

Metals used in surface plasmon resonance biosensors are gold $\left(\varepsilon_{\mathrm{m}}=-11.55+\mathrm{j} 1.28\right.$ at $630 \mathrm{~nm}[16])$ and silver $\left(\varepsilon_{\mathrm{m}}=-18.10+\mathrm{j} 0.50\right.$ at $630 \mathrm{~nm}$ [16]). However, research is also focused on discovering new multilayer metal films to increase the sensitivity of sensors [17]. Gold is often preferred to other metals as it is chemically more stable and can therefore be used for a longer period before being replaced. 


\subsection{Surface Plasmon Coupling}

For surface plasmon propagation to occur, the incident light must be coupled to the interface. The most obvious would be to excite the surface plasmon from the dielectric medium; however this is not possible as the momentum of the photons is not great enough to excite a surface plasmon. This is shown on Figure 2.2 where the solid blue line (photon) never meet the dashed blue line (surface plasmon) since the momentum of the surface plasmon will always be larger than the momentum of the photon at all wavelengths when they both travel through the same medium. There are three main ways to couple light: prism coupling, grating coupling and waveguide coupling.

In order to solve the mentioned issue using prism coupling, the photons must travel through a high refractive index medium on the metal side opposite the dielectric medium. It is possible to excite a surface plasmon from this side of the metal given that the metal film is thin enough to allow light to go through. As the penetration depth of light in metal such as gold and silver is around $100 \mathrm{~nm}$ [18], if the film is thinner than that the excitation will be possible as long as the refractive index of the medium is higher than that of the dielectric medium where the surface plasmon propagates. Glass is often used for this purpose. A shown in Figure 2.2, the red lines represent the propagation constants of the photon and plasmon in SF11 glass and the photon momentum in glass equals the surface plasmon momentum in water at a specific wavelength which therefore means that the conditions for surface plasmon resonance are met. 


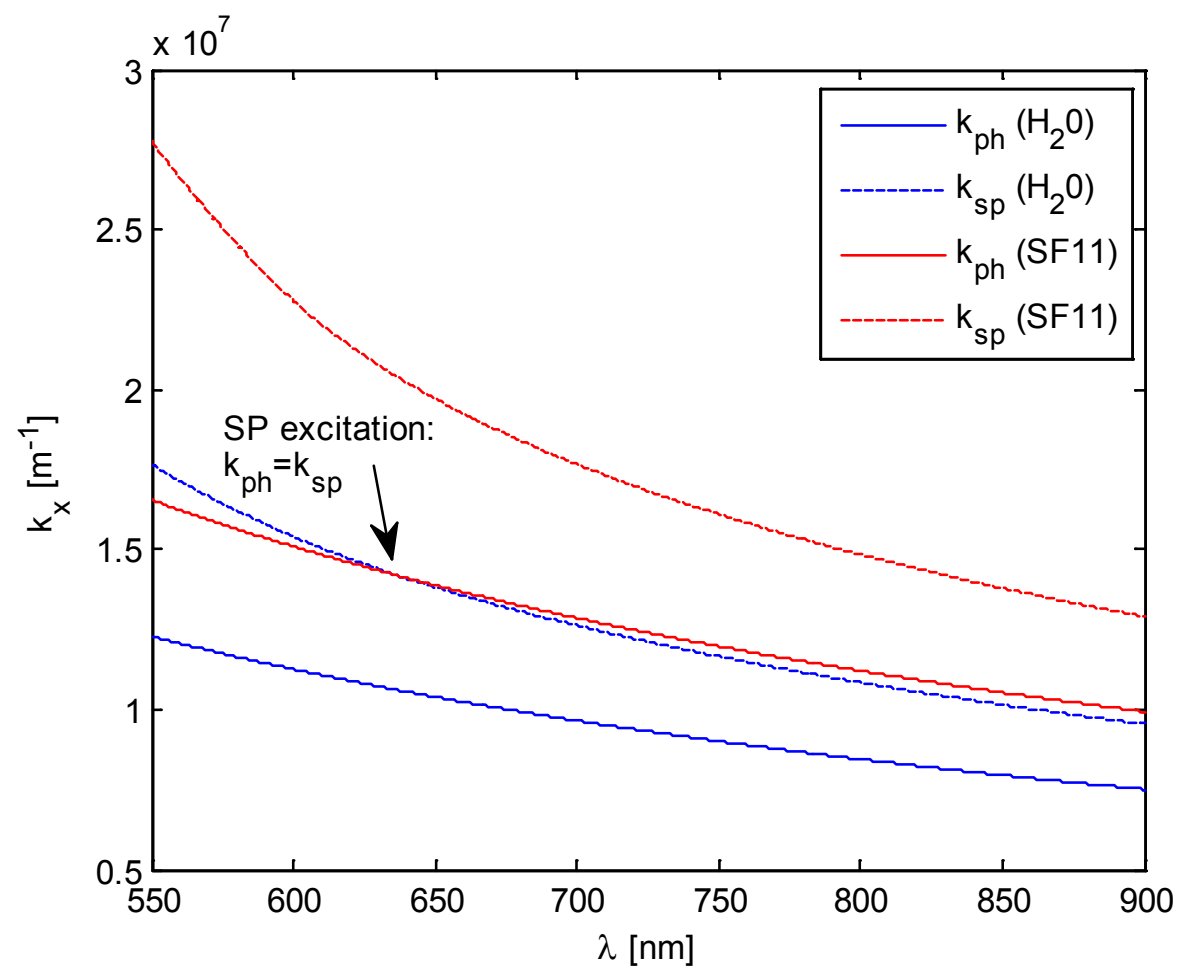

Figure 2.2 - Propagation constant, $k_{x}$, of the photon $\left(k_{p h}\right)$ and the surface plasmon $\left(k_{\mathrm{sp}}\right)$ in water $(n=1.33)$ and glass $(\mathrm{SF11}-\mathrm{n}=1.78)$ using a $50 \mathrm{~nm}$ gold metal layer (parameters from [16]) and an incident angle of 54.

This coupling method with a glass prism placed on the metal side opposite the dielectric medium to enhance the photons momentum is called attenuated total reflection (ATR). There are two possible configurations: Kretschmann and Otto. In the Kretschmann configuration (Figure $2.3 \mathrm{a}$ ), the prism is directly in contact with the metal while in the Otto configuration (Figure $2.3 \mathrm{~b}$ ), the dielectric medium is between the prism and the metal. The most popular ATR method is the Kretschmann configuration. This is the case mainly because the Kretschmann configuration is easier to implement than the Otto configuration since the glass is adjacent to the metal so that the thin metal film can be deposited on the glass while for the Otto configuration, the sample, which is liquid or gaseous, is between the glass and the metal film. It is challenging to create a small liquid chamber covered by a flat film a few nanometres thick. 

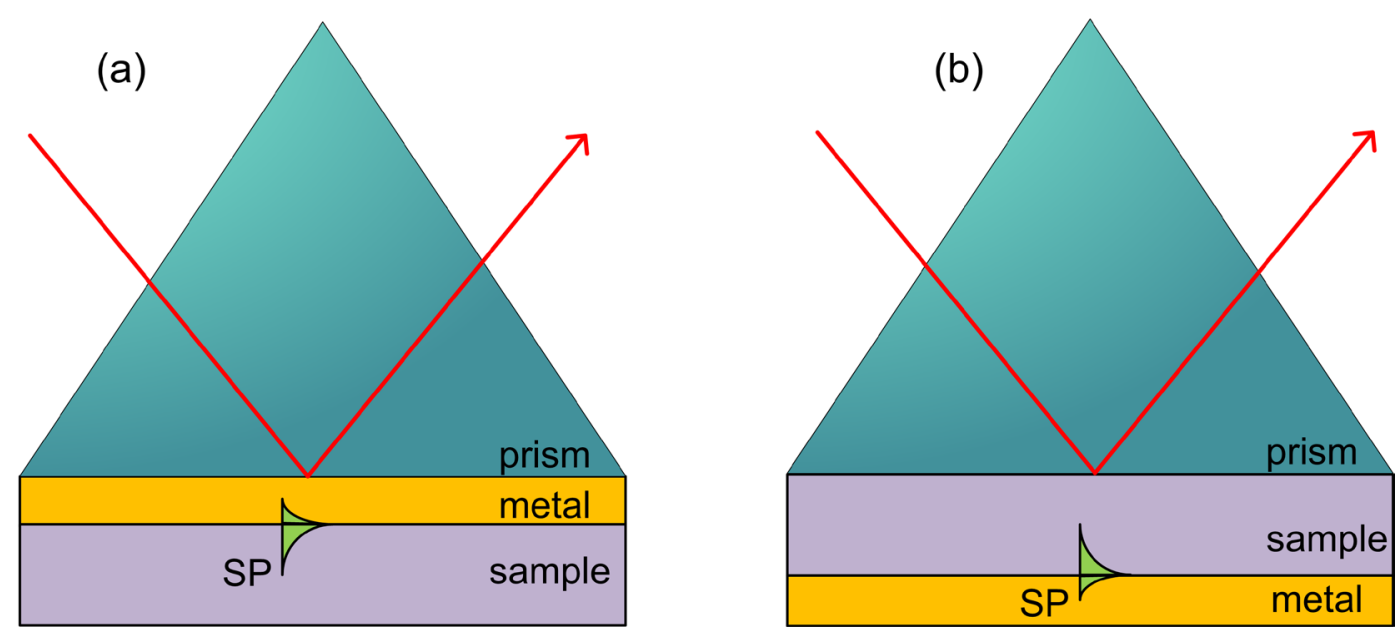

Figure 2.3 - ATR coupling methods: (a) Kretschmann configuration, (b) Otto configuration

The most frequently used glasses for ATR coupling are N-BK7 $\left(n_{p}=1.51509\right.$ at $632.8 \mathrm{~nm}$ [19] $)$ and N-SF11 $\left(\mathrm{n}_{\mathrm{p}}=1.77860\right.$ at $632.8 \mathrm{~nm}$ [19]). The propagation constant of the photons depends on the polarisation as well as the angle of incidence, $\theta$, as only the component parallel to the metal surface and along the propagation direction can excite surface plasmons. The propagation constant of the photons is therefore:

$$
k_{p h}=\frac{2 \pi}{\lambda} n_{p} \sin \theta
$$

Although the prism-based coupling is the most widely used with surface plasmon resonance biosensing, there are two others ways of coupling light: grating coupling and waveguide coupling. Grating coupling consists in coupling light to a surface plasmon through the sample by using a periodically corrugated metal surface as shown on Figure 2.4. A collimated beam from a broadband source is incident on a grating and the reflected light is measured for one of the orders with a photodetector array. The grating enhances the photons momentum and the light is coupled into a surface plasmon at a specific wavelength. 


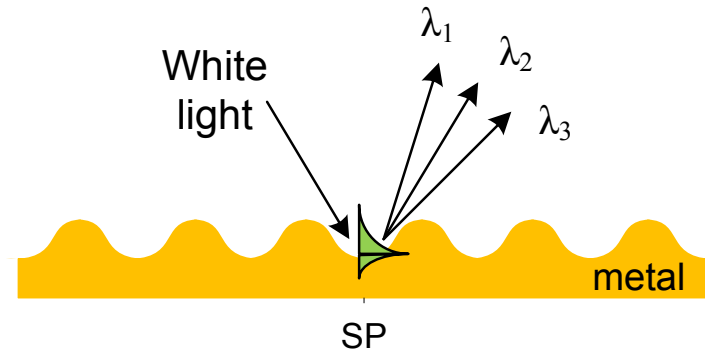

\section{Figure 2.4 - Grating coupling}

Surface plasmon coupling can be done through waveguides as well. In this case, the input light is introduced in a waveguide and a guided mode propagates in the core. The part of the mode propagating in the cladding excites a surface plasmon when it hits a metal section in the cladding. Then the surface plasmon propagates along the interface between the metal and the surrounding dielectric medium when the propagation constant of the guided mode in the cladding is equal to the one of the surface plasmon [20]. Figure 2.5 illustrates this concept. The advantages and disadvantages of these three coupling methods will be stated in Chapter 3.

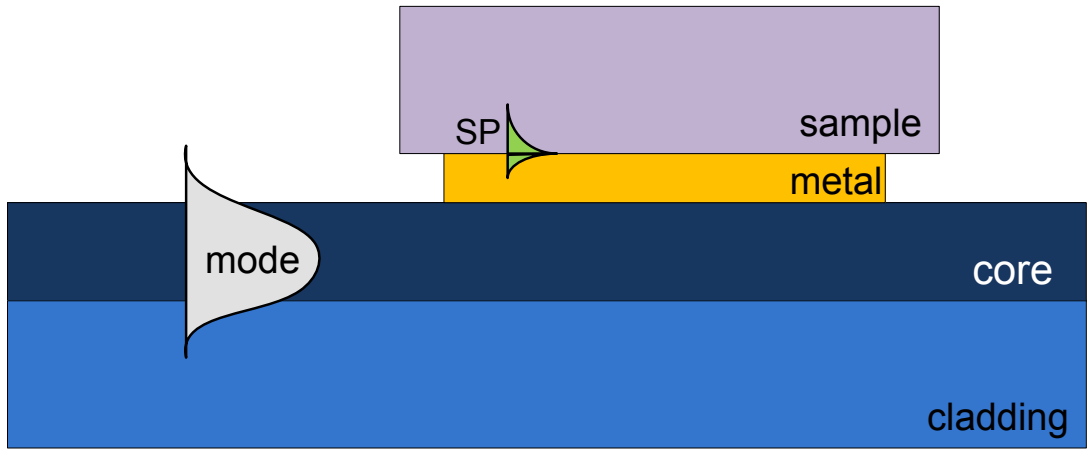

Figure 2.5 - Waveguide coupling 


\subsection{Surface Plasmon Resonance}

Transverse-magnetic polarised light must be used in order to excite surface plasmons (see Figure 2.6). TM polarised light has the electric field components $E_{x}$ and $\mathrm{E}_{\mathrm{z}}$ and the magnetic field component $\mathrm{H}_{\mathrm{y}} ; \mathrm{z}$ being perpendicular to the surface interface and $\mathrm{x}$ and $\mathrm{y}$ being parallel to the surface with $\mathrm{x}$ in the direction of the surface plasmon's propagation. Therefore, transverse-electric (TE) polarised light would not excite surface plasmons as it does not have an $\mathrm{x}$ electric field component. However, it can still be useful for normalisation or referencing to reduce the noise in the data.

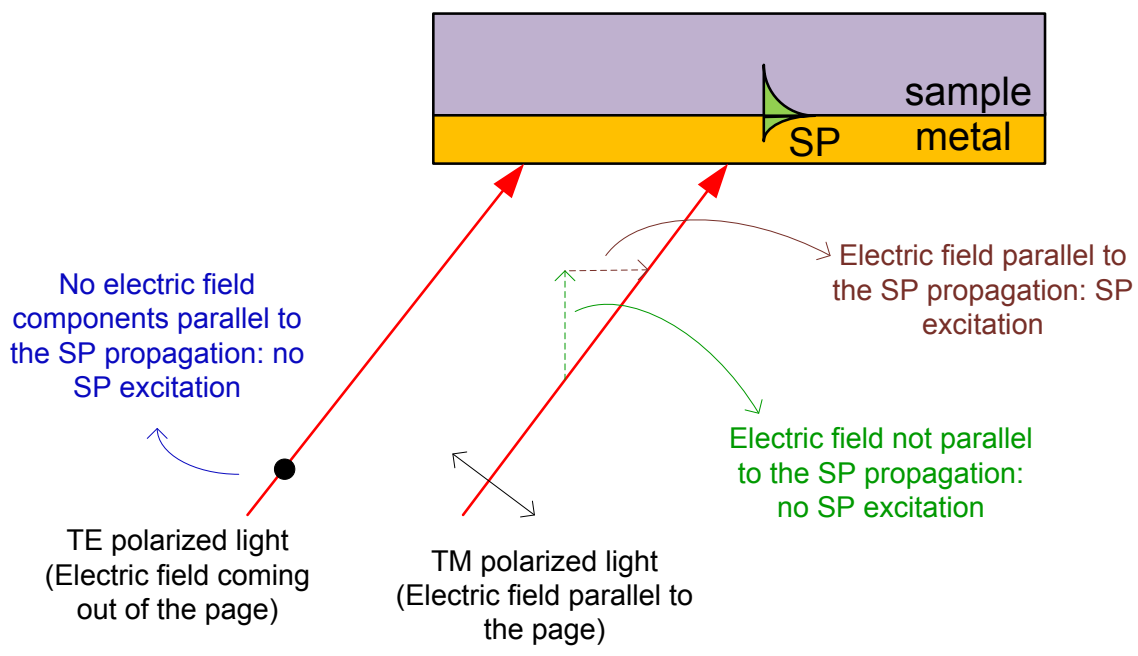

Figure 2.6 - Excitation of surface plasmon by TM and TE polarised light

The surface plasmons are excited when the incident photons momentum is equal to the surface plasmons momentum. When this happens, the light incident on the metal does not reflect and the energy is transferred to the surface plasmons allowing them to propagate. This phenomenon can be observed by measuring the reflected light. The light is totally reflected unless the resonance conditions are met; then there is no reflected light. Using one varying parameter, either angle of incidence or wavelength, the light reflected can be measured to obtain a curve of 
the intensity versus the varying parameter revealing a dip at the resonant angle or wavelength as shown in Figure 2.7.

In Figure 2.7, the resonant conditions occur at $\theta=54.6^{\circ}$ as it can be seen from the absence of reflection. This SPR curve is very important since tracking the minimum position corresponds to measuring refractive index change which is the basis of biosensing. The asymmetry in the curve is due to total internal reflection which occurs around $48^{\circ}$ and creates a hook shape in the reflectance curve.

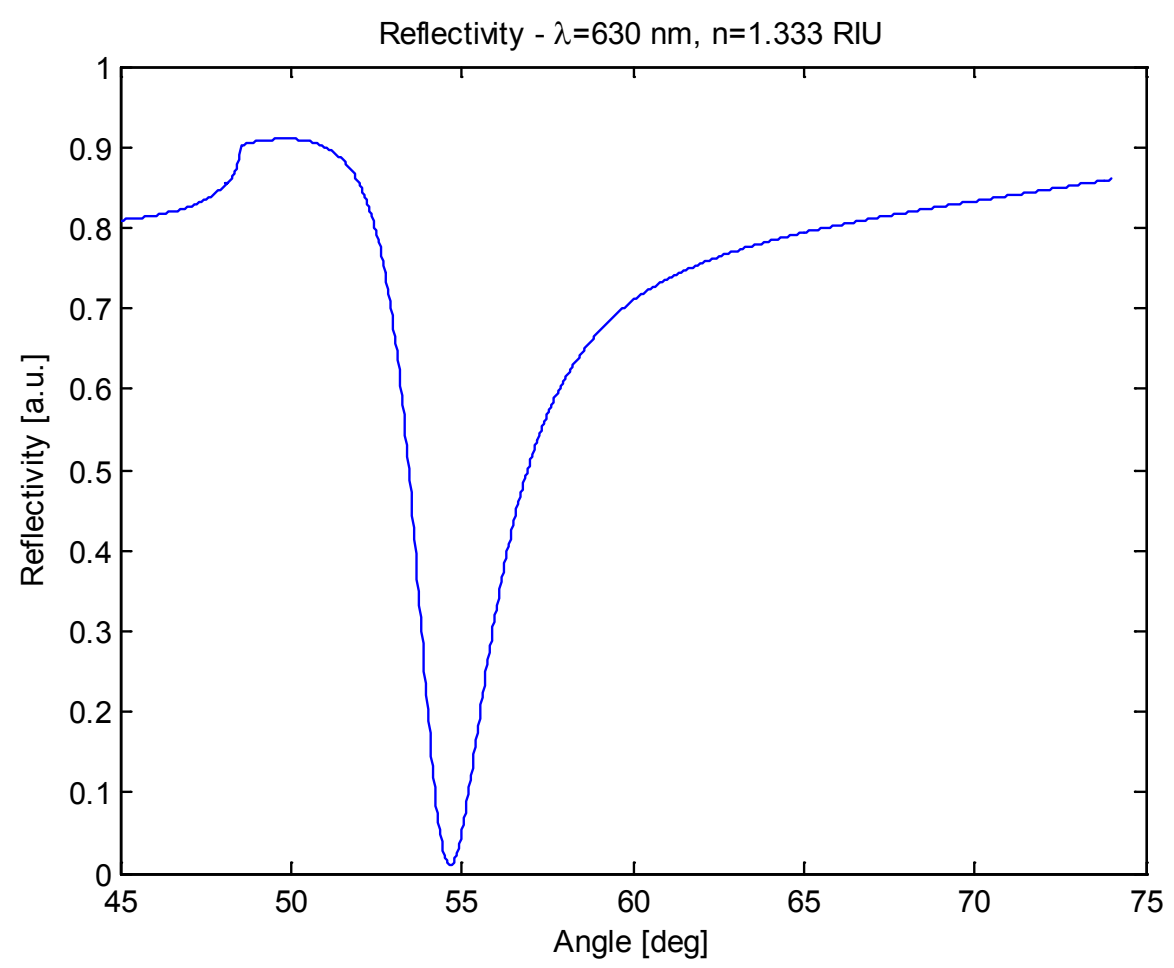

Figure 2.7 - Reflected light with respect to angle of incidence at a wavelength of $630 \mathrm{~nm}$ with a dielectric refractive index of 1.333 refractive index unit (RIU).

A general equation for the reflectance with respect to the different parameters can be used in order to find the resonance conditions. This equation is obtained by using Fresnel's equations. The reflectance, R, for a three layer system, 0/1/2 (e.g.: prism/metal/dielectric medium), is [21]: 


$$
\begin{gathered}
R=\left|r_{012}\right|^{2}=\left|\frac{E_{r}}{E_{0}}\right|^{2}=\left|\frac{r_{01}+r_{12} \exp \left(2 i k_{z 1} d\right)}{1+r_{01} r_{12} \exp \left(2 i k_{z 1} d\right)}\right|^{2} \\
r_{i k}=\left(\frac{k_{z i}}{\varepsilon_{i}}-\frac{k_{z k}}{\varepsilon_{k}}\right) /\left(\frac{k_{z i}}{\varepsilon_{i}}+\frac{k_{z k}}{\varepsilon_{k}}\right) \\
k_{z i}=\left[\varepsilon_{i}\left(\frac{2 \pi}{\lambda}\right)^{2}-k_{x}{ }^{2}\right]^{1 / 2}
\end{gathered}
$$

The reflectance $R$ is the overall ratio of the reflected electric field, $E_{r}$, to the incident electric field, $E_{0}$, squared. $r_{i k}$ is the ratio of reflected electric field with respect to the incident electric field on the interface between layers $i$ and $k, \mathrm{k}_{\mathrm{zi}}$ is the propagation constant in the $\mathrm{z}$ direction in layer $i$ and $\mathrm{k}_{\mathrm{x}}$ is the input propagation constant which in this case is equal to $k_{\mathrm{ph}}$ (equation 2.4). Finally, $\mathrm{d}$ is the middle layer (metal)'s thickness. The angle of incidence, $\theta$, is found in the equation for $\mathrm{k}_{\mathrm{ph}}$ and the wavelength is found in $\mathrm{k}_{\mathrm{zi}}$ as well as in the electrical constants of the metal and prism. Using equations 2.5 to 2.7 the SPR curve can be simulated for any chosen parameters.

\subsection{Interrogation Methods for Prism-Based Coupling}

There are multiple ways to interrogate a sample with SPR via prism coupling: angular, wavelength, intensity and phase (interference) interrogations. Figure 2.8 shows those different methods. Angular and spectral interrogations are the most often used ones as they are very sensitive methods for a relatively simple design to implement. For angular interrogation, a monochromatic beam of light is incident on the metal at different angles to obtain an intensity versus angle curve. For wavelength interrogation, a broadband source shines at a fixed angle on the metal to obtain an intensity versus wavelength curve. In those two methods, the curve is similar and shows a dip at the resonant angle/wavelength (Figure 2.7). 
Intensity modulation does not have a sensitivity as great as the other methods. However, it is still quite often used as it is well suited for multichannel sensing and is therefore a useful tool for imaging. In this case, a monochromatic light is used at a fixed angle close to the resonant angle and the difference in intensity is measured as the refractive index changes in the sample. This method is convenient for imaging since the source is fixed in angle and wavelength and a large area can be covered by the input beam; thus a large number of sensing channels can be measured at the same time.

Finally for phase detection or interferometric SPR, the phase difference between the input source and the reflected light is measured at a fixed wavelength and fixed resonant angle by interference. The phase of the reflected beam changes with respect to the incoming beam as the refractive index changes therefore permitting a phase different to be measured. Phase detection can have a better resolution than spectroscopy but the design is slightly more complicated and the main disadvantage of this method is the poor dynamic range. The interferometer SPR biosensors are highly sensitive but only close to the inflection point of the phase versus refractive index curve as seen on Figure 2.9. The steepness of the curve allows a large sensitivity but it simultaneously greatly limits the dynamic range.

Additionally to those methods the first two presented can be combined to create another interrogation method: spectro-angular SPR measurements. In this case, the reflectivity is measured both for a range of angles and a range of wavelengths. This is the method used for the biosensor in this thesis and it will be detailed in Chapter 4. 

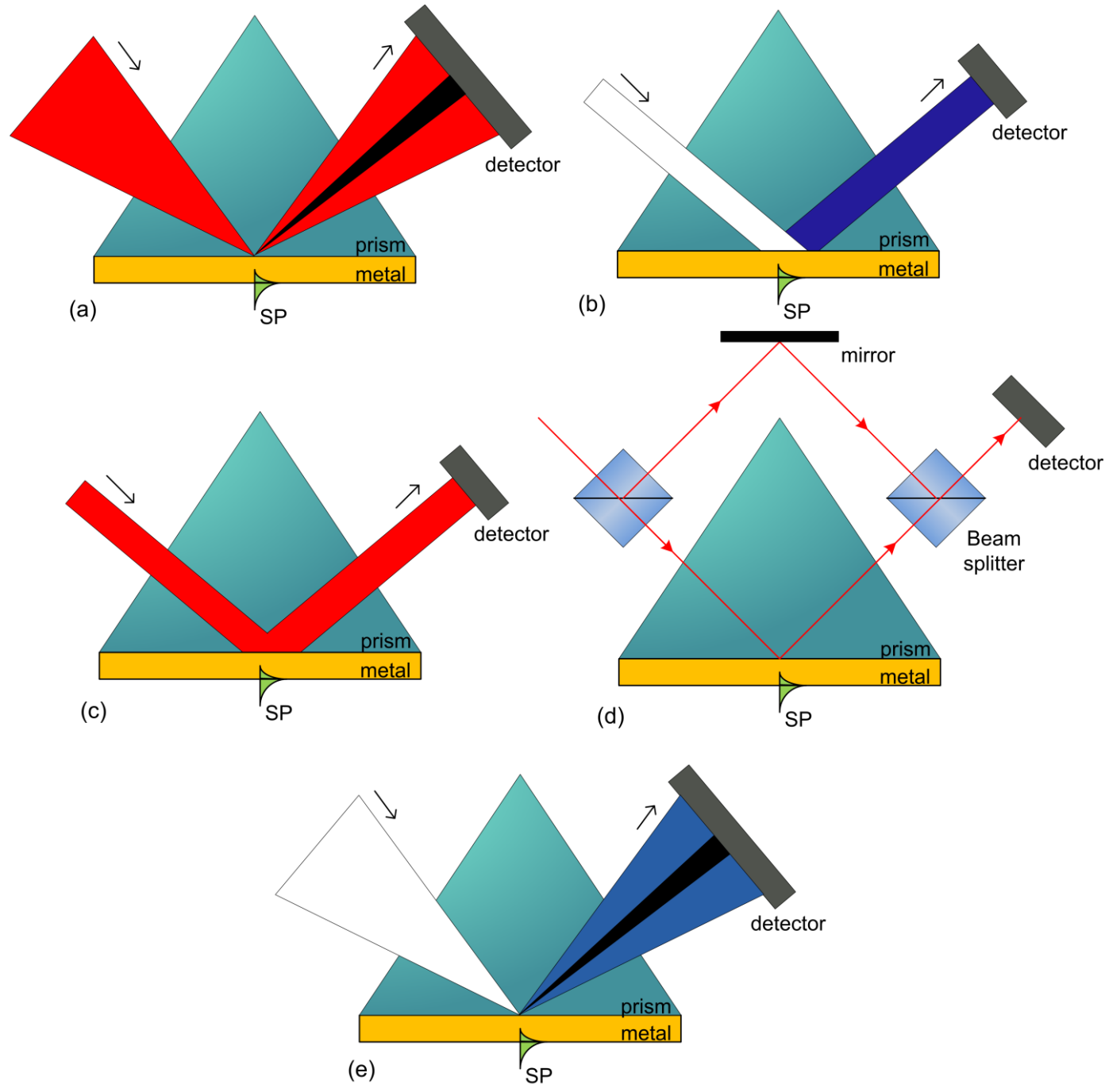

Figure 2.8 - Five different methods of interrogation: (a) angular, (b) wavelength, (c) intensity (d) interferometric and (e) spectro-angular. 


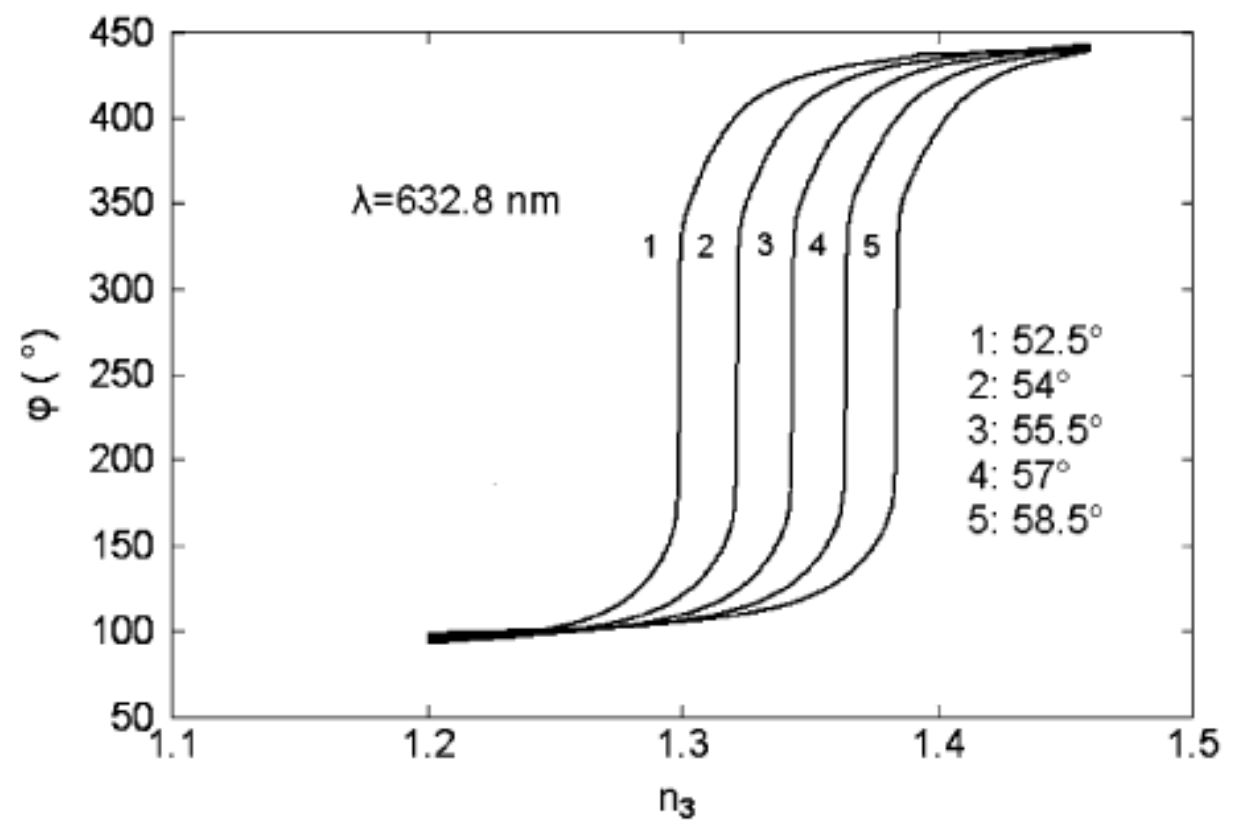

Figure 2.9 - Phase shift with respect to refractive index for different angle of incidence [22]. The dynamic range in this case is 0.002 RIU.

\subsection{Refractive Index Change Measurement With SPR}

Surface plasmon resonance is one of the most widely used methods for biosensing. It can be used to accurately measure a difference in the refractive index of a sample since the reflectance response is dependent upon the dielectric medium's refractive index. Choosing an interrogation method, the shift in the resonance conditions can be seen as the refractive index changes. Figure 2.10 shows the shift occurring in the SPR curve with a change in refractive index from 1.3 to 1.4 RIU for angular interrogation.

For both angular and wavelength interrogation, the change in refractive index is measured by finding the difference in the resonant conditions, either the angle of incidence or the wavelength. The sensitivity can be measured by calculating the angular shift in degrees for 1 RIU. Table 2.1 gives the sensitivity at $630 \mathrm{~nm}$ and $850 \mathrm{~nm}$ considering two metal options, gold $(\mathrm{Au})$ and silver $(\mathrm{Ag})$, and two glass 
options, SF11 and BK7. Another factor considered on the SPR curve is the full width at half maximum (FWHM); the smaller the width, the sharper the curve. A sharper curve is better as it is easier to find the resonant condition with the dipfinding algorithm. The figure of merit is sometimes used as well a comparison between sensors. It is a ratio of the sensitivity to the width of the SPR curve [23].

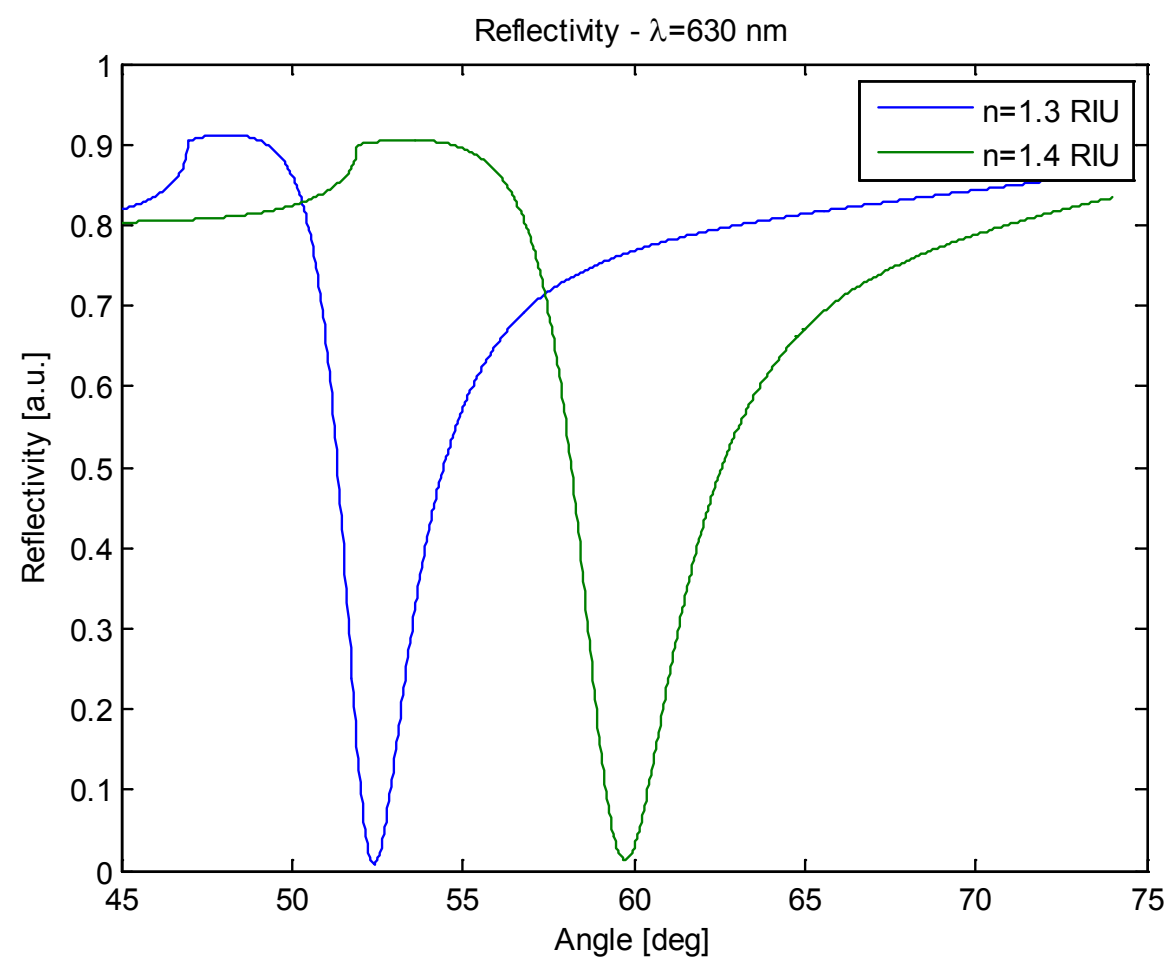

Figure 2.10 - Shift in the resonance conditions (incident angle) reflecting the change in refractive index of the sample at a wavelength of $630 \mathrm{~nm}$.

As it can be seen from the data in Table 2.1, the sensitivity is better with gold than silver, with BK7 than SF11 and at $630 \mathrm{~nm}$ than $850 \mathrm{~nm}$. For the SPR curve, it is much sharper with silver than gold, with SF11 than BK7 and at $850 \mathrm{~nm}$ than 630 $\mathrm{nm}$. The conclusion might be made that as the SPR dip is sharper the sensitivity is lower. However, it is not as true in experimental set-up as even if the angular shift is greater per refractive index at $630 \mathrm{~nm}$, the sharpness of the curve at $850 \mathrm{~nm}$ is much easier to analyse in the presence of noise. 
Table 2.1 - Theoretical sensitivity and FWHM of the SPR curve for $630 \mathrm{~nm}$ and $850 \mathrm{~nm}$ for angular SPR measurement. The metals have a thickness of 50 $\mathrm{nm}$ and the refractive index of the dielectric is around 1.33. The values for gold and silver are taken from [16] and the values for SF11 and BK7 are from [19].

\begin{tabular}{|l|c|c|c|c|}
\hline Glass / Metal & \multicolumn{2}{|c|}{630 nm } & \multicolumn{2}{c|}{850 nm } \\
& Sensitivity & FWHM & Sensitivity & FWHM \\
& $\left({ }^{\circ} /\right.$ RIU) & $\left({ }^{\circ}\right)$ & $\left({ }^{\circ} /\right.$ IU) & $\left.{ }^{\circ}\right)$ \\
\hline $\mathrm{SF} 11 / \mathrm{Au}$ & 70 & 3.5 & 57 & 0.7 \\
\hline $\mathrm{SF} 11 / \mathrm{Ag}$ & 61 & 0.6 & 56 & 0.2 \\
\hline $\mathrm{BK} 7 / \mathrm{Au}$ & 150 & 6 & 101 & 1.2 \\
\hline $\mathrm{BK} 7 / \mathrm{Ag}$ & 116 & 1 & 96 & 0.3 \\
\hline
\end{tabular}

\subsection{Short-Range SPR, Long-Range SPR and Localized SPR}

The surface plasmon propagating on a metal-dielectric interface as presented so far is also called the short-range surface plasmon or asymmetric surface plasmon. These terms are used when a distinction is required between the short-range surface plasmon and the long-range surface plasmon or symmetric surface plasmon. Long-range surface plasmons are TM waves that propagate symmetrically on both interfaces of a thin metal film when the dielectric media on both sides are of equal refractive indices. The long-range surface plasmon is used in certain applications, such as nonlinear optics, because its propagation length along the interface is much longer than short-range surface plasmon since the attenuation is a least 2 to 3 times lower [24]. Short-range SPR is most often used in biosensing applications due to its larger sensitivity but long-range SPR can be valued in applications where the longer propagation length can overall be beneficial for example for fluorescence-based sensing. 
The other way surface plasmon resonance can be used in biosensing is called localized surface plasmon resonance (LSPR). In the conventional metal-dielectric interface, the EM waves are confined to the boundary since they decay exponentially in both media and therefore only the electrons at the metal surface are excited. On the other hand, localized surface plasmons are collective oscillations of the conduction electrons in metal nanoparticles (Np) [25]. Figure 2.11 shows a localized surface plasmon and the difference with a propagating surface plasmon can be seen by comparing this figure with Figure 2.1. Localized surface plasmon resonance happens when the nanoparticles are much smaller than the incident wavelength and thus the electric field propagating around them is quasi-static. The wavelength of the surface plasmons is dependent on the refractive index of the immediate dielectric around the nanoparticles. Therefore, they are sensitive to the change in the refractive index of the dielectric and can be used for biosensing applications. LSPR can also be combined with SPR to achieve an enhancement of the SPR response and increase the sensitivity of biosensors [26]. For the work in this thesis, only prism-based short-range surface plasmon resonance is considered for biosensing.

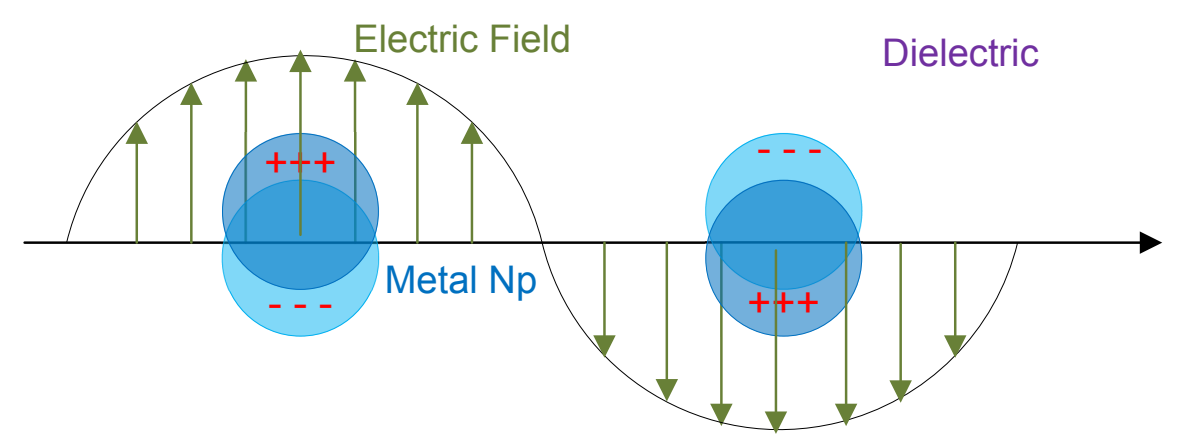

Figure 2.11 - Localized surface plasmon 


\section{Chapter 3: BIOSENSING}

\subsection{Fundamentals of Biorecognition}

The ultimate goal of a biosensor is to detect and measure analyte of interest. The analyte are the target biomolecules; in medical and environmental applications, they can be proteins, contaminants, vitamins, drugs, etc. SPR is the most widely used method to implement biosensors as it is well suited to perform the detection and the concentration measurements. As shown in the previous chapter, SPR allows a change in the refractive index of a sample to be observed. The refractive index change is linked to the concentration of biomolecules, the greater the refractive index change the higher the concentration of analyte (in a specific buffer). Obtaining the SPR measurement of a buffer before and after adding biomolecules will give information on the concentration of biomolecules.

A biosensor has two main implementation steps: the binding and the transduction mechanism. The binding is the immobilization of analyte of interest on the sensing surface and it will be presented in this chapter. A transduction mechanism is a way of translating the presence of analyte into a measurable signal. In the case of SPR biosensor, the surface plasmon propagation with the reflectivity measurement is the transduction mechanism. The SPR curve obtained in response of the binding can be analysed to give information about the sample.

In order to have biorecognition, the interrogated analyte must be fixed on the metal surface. It is important that the molecules are very close to the surface because the electric field of the surface plasmon decays exponentially in the medium and can only sense the region close to the surface. This region is limited by the decay of the field and is the distance where the amplitude is 1/e times the maximum amplitude [18] (equation 3.1). 


$$
z_{d}=\frac{1}{\left|k_{z d}\right|}
$$

$\mathrm{Z}_{\mathrm{d}}$ is the thickness of the sensing region and $\mathrm{k}_{\mathrm{zd}}$ is the propagation constant in the $\mathrm{z}$ direction in the dielectric medium. The propagation length of the surface plasmon along the interface is also an important factor to consider and is given by equation 3.2. Table 3.1 gives the propagation length and field depth in the dielectric medium for gold and silver at $630 \mathrm{~nm}$ and $850 \mathrm{~nm}$ and Figure 3.1 shows the decaying fields in both gold and water across their interface.

$$
L=\frac{1}{2\left|k_{s p}^{\prime \prime}\right|}
$$

Table 3.1 - Theoretical propagation length, $L$, and sensing region thickness, $z_{d}$, for $630 \mathrm{~nm}$ and $850 \mathrm{~nm}$. The metals, gold and silver, have a thickness of 50 $\mathrm{nm}$ and the refractive index of the dielectric is $\mathbf{1 . 3 3}$. The values for gold and silver are taken from [16].

\begin{tabular}{|l|c|c|c|c|}
\hline Metal & \multicolumn{2}{|c|}{$630 \mathbf{n m}$} & \multicolumn{2}{c|}{$850 \mathbf{~ n m}$} \\
& $\mathbf{L}(\boldsymbol{\mu m})$ & $\mathbf{z}_{\mathbf{d}}(\mathbf{n m})$ & $\mathbf{L}(\boldsymbol{\mu m})$ & $\mathbf{z}_{\mathbf{d}}(\mathbf{n m})$ \\
\hline Gold & 3 & 178 & 24 & 394 \\
\hline Silver & 25 & 229 & 141 & 445 \\
\hline
\end{tabular}

As it can be seen from the data in Table 3.1, the propagation length and the sensing region are longer for silver than for gold and for $850 \mathrm{~nm}$ than for $630 \mathrm{~nm}$. Additionally, the SPR curve is much sharper for silver than for gold and for 850 $\mathrm{nm}$ than for $630 \mathrm{~nm}$. It suggests that silver is a better option than gold for biosensing since it can allow the plasmon to sense a larger region and a sharper SPR curve improves the data analysis and thus the precision of the refractive index change. However, gold is more often used as it has a better long-term stability than silver. 


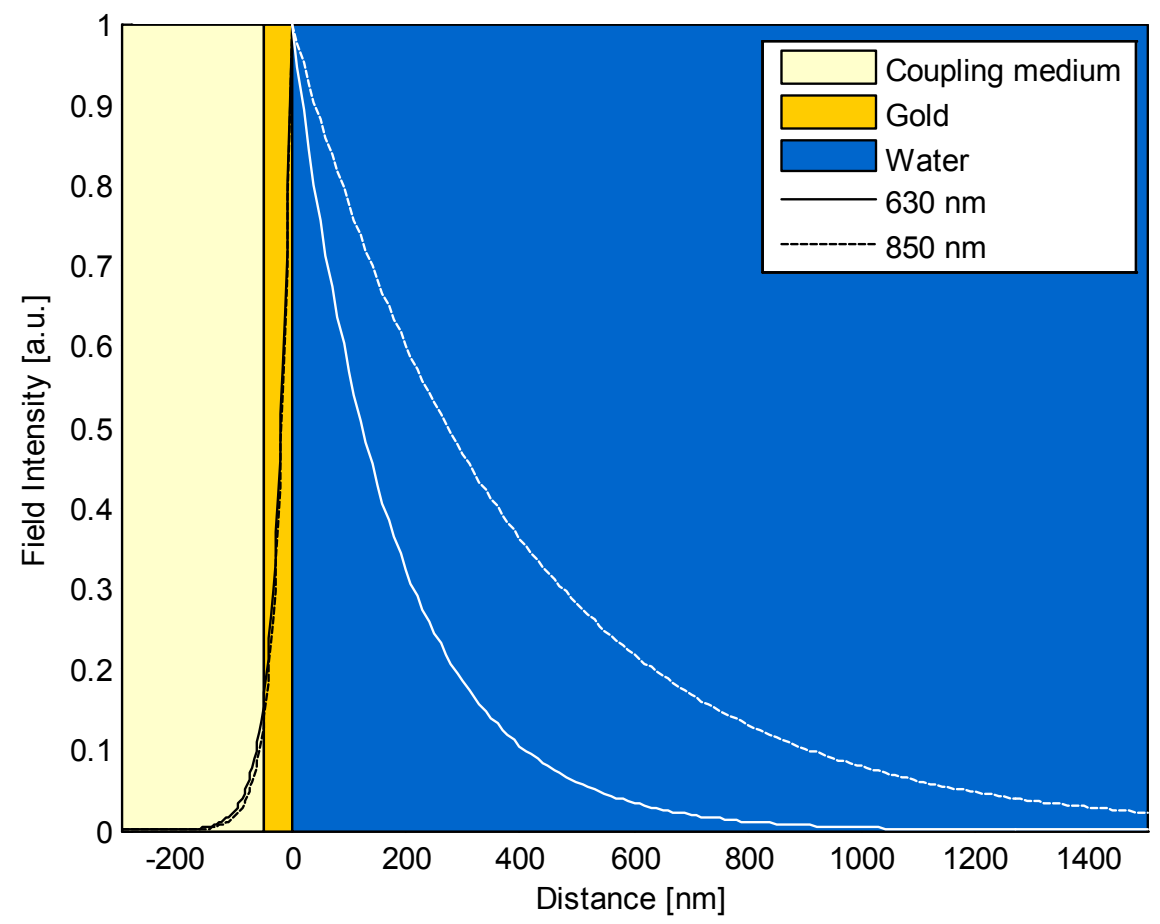

Figure 3.1 - Decaying fields in gold $(50 \mathrm{~nm})$ and water $(\mathrm{n}=1.33)$ for $630 \mathrm{~nm}$ and $850 \mathrm{~nm}$.

In order to make an accurate measurement, only the analyte wanted must be present in the sample. This is done by using a biological recognition element (BRE). A BRE is an element that can recognize another one by specific binding and antibodies are the most often used BRE [20]. The BRE will first be introduced in the sensing region in the buffer and will be immobilized on the sensing surface. Then the analyte are introduced and they will bind to the BRE while any other types of molecules will flow by and be rejected (see Figure 3.2). Any non-specific binding would produce incorrect results, thus the choice of BRE is crucial to the performance of the biosensor. The immobilization of analyte improves the specificity of the measurement. Even though the BRE are chosen for the target analyte, some weak binding will happen with other biomolecules. Using a detergent to wash the excess biomolecules will clear the sample from nonspecific binding while the target analyte will be strongly bound to the BRE and will stay on the surface. 


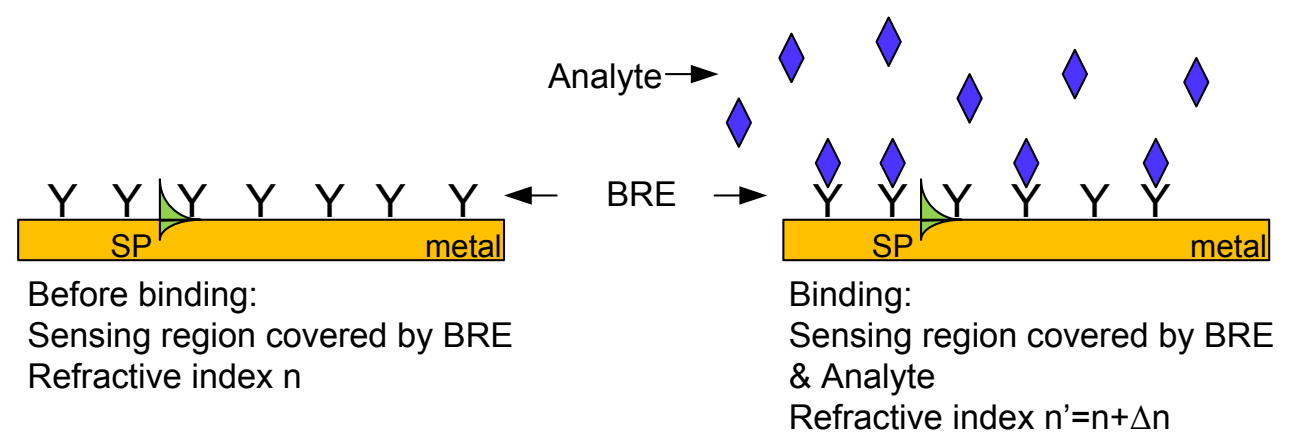

Figure 3.2 - Sensing surface with BRE (left) and binding of analyte to the BRE (right).

As shown in Figure 3.2, the SPR measurements are taken before and after the binding of analyte which results in a differential refractive index measurement $(\Delta \mathrm{n})$. There is no label required in this assay, therefore making the SPR method label-free. In this method, the analyte binding changes the refractive index of the sample at the surface which results in a measurable change in the output. For labelling, a second antibody attached to a luminescent particle would be required to create the output measurement. This method increases the complexity and the cost of the process. Additionally, the label can affect the binding of the analyte and modify the measurements of association and dissociation constants.

There are different ways to enhance the SPR response at the chemistry level in a biosensor. It involves the improvement of the sensitivity by increasing the magnitude of the output signal for a given concentration of analyte by adding a label. One way is to introduce a second group of antibodies after the binding to form a sandwich assay, antibody-analyte-antibody [18]. The second set of antibodies will increase the refractive index change and the SPR response linearly with respect to the concentration of analyte. Another method of SPR signal enhancement is to attach nanoparticles to the analyte [26]. In this case, nanoparticles bind to the analyte or to the second set of antibodies (in the case of a sandwich assay) and enhance the SPR response through localized surface plasmon 
resonance and the interaction between the localized surface plasmon and the propagating surface plasmon. These are interesting options when it comes to improving the sensitivity of the biosensor but the drawback is that the biosensor is not label-free anymore.

\subsection{Sensitivity and Resolution}

There are two principal characteristic used to evaluate and compare the performance of different biosensors: the sensitivity and the resolution. The sensitivity is a measure of the change in the output of the sensor, Y, with respect to changes in the input conditions, c. For SPR biosensor, the output of the sensor is the resonance angle or the resonance wavelength, etc and the change in the input conditions corresponds to the concentration of analyte. The sensitivity is given by the following [27]:

$$
S_{C}=\frac{\partial Y}{\partial c}
$$

This equation can be divided in a product of two ratios. The first is the sensitivity of the output to the change in the index of refraction, $\mathrm{S}_{\mathrm{RI}}$, and the second is the sensitivity of the index of refraction to the change in the concentration of analyte, $\mathrm{S}_{\mathrm{nc}}$. The sensitivity equation can be rewritten as follow [27]:

$$
S_{C}=\frac{\partial Y}{\partial c}=\frac{\partial Y}{\partial n} \cdot \frac{\partial n}{\partial c}=S_{R I} S_{n c}
$$

The objective is to obtain the highest sensitivity possible so that a small change in the concentration of analyte produces a large output shift. The $S_{\mathrm{nc}}$ ratio can be increased by using labels such as a secondary antibody as mentioned before. Otherwise for a direct assay, this ratio is fixed. The value of the $\mathrm{S}_{\mathrm{RI}}$ ratio depends on the chosen interrogation method, on the materials used and the choice of parameters. 
The resolution, or the lowest detection limit, is the smallest possible change in a sample's refractive index that produces a measurable change in the output. It is given in refractive index unit (RIU). This measure depends on the noise level in the output of the sensor. The resolution, $\sigma_{\mathrm{RI}}$, can be expressed in terms of the standard deviation of noise in the sensor's output, $\sigma_{N}[28]$ :

$$
\sigma_{R I}=\sigma_{N} / S_{R I}
$$

The objective is to have the smallest resolution possible. The sensor's resolution can be mapped into the smallest measurable concentration of a specific analyte. Most of the research being done on biosensors focuses on improving the detection limit since a lower resolution allows diagnosis to be made earlier. In this case, the main challenges are to improve the quality of the data collected by the detectors and improve the data processing. Most of the noise comes from the detectors and a good data analysis can reduce the effects on the results.

\subsection{Advances in SPR Biosensors}

Research is being conducted to improve the sensitivity and detection limit of biosensors and surface plasmon resonance is one of the most widely used methods for implementing biosensors. SPR sensors today are reaching very low detection limit and as the research is still focused on increasing the performance, other characteristics of the sensors are put forward such as portability, high throughput and real-time monitoring. SPR biosensors are available commercially and the best resolution to this date is $10^{-7}$ RIU and it can be achieved with a Biacore SPR biosensor [20,29]. For the different coupling methods and interrogation methods presented here, the detection limit achieved varies and each method has its advantages and disadvantages. 


\subsubsection{Prism-based Coupling}

Sepúlveda et al. proposed an intensity modulation SPR biosensor using the magneto-optic effect of metal by designing a multilayer metal film [17]. It enhanced the sensitivity of the biosensor with respect to conventional intensitybased SPR biosensors and they obtained a detection limit of $5 \times 10^{-6}$ RIU. As mentioned earlier, the great advantage of intensity modulation is that it can be used for imaging a surface. If a surface area is illuminated rather than a point, each pixel of a detector will be picking up a different intensity that will depend on the refractive index of a specific spot on the surface which in the end will give an image of the surface. Piliarik et al. showed the measurement of 64 independent channels simultaneously with a refractive index resolution of $2 \times 10^{-6}$ RIU [30]. They demonstrated the use of their sensors for the detection of oligonucleotides.

Although SPR imaging is an important asset, intensity modulation does not offer a detection limit as low as spectroscopy. Angular and spectral interrogations are the most widely used method for highly sensitive biosensing. Nenninger et al. demonstrated an SPR biosensor based on wavelength modulation. They performed an analysis of the source of noise in the output of SPR sensors and concluded that the detector is the dominant source of noise. They obtained a limit of detection of $2 \times 10^{-7}$ RIU with their experimental set-up which corresponded to their theoretical analysis [31]. Slavík and Homola reported an even more sensitive device. The sensor was based on long-range SPR and used wavelength interrogation. They obtained a resolution of $2.5 \times 10^{-8}$ RIU [32].

SPR biosensors based on interferometry can achieve a sensitivity that is equally as good or better than that of angular or spectral interrogations. Nikitin et al. developed an interferometric biosensor and used it for an imaging as well as a sensing device. The resolution of their system was $10^{-7}$ RIU [33]. Kong also used a Mach-Zender interferometer design to fabricate an SPR biosensor. The phase was measured for both polarisations state; only p-polarised light excite surface 
plasmon so the s-polarised signal was used as a reference. The noise reduction performed by the referencing allowed the resolution to be as low as $5.5 \times 10^{-8}$ RIU [34]. Although the sensitivity is great in these sensors, the lack of dynamic range can sometimes be a drawback. A very small dynamic range means that the biosensor can be used only for samples with selected refractive indices. This will reduce the applications of the sensor as it will have to be design for the detection of a specific analyte.

\subsubsection{Grating Couplers}

Instead of using a glass prism for coupling surface plasmons, a diffraction grating can be used. This is what Dostálek et al. did for their SPR biosensor. They holographically fabricated sinusoidal gratings to develop a multichannel sensor. Their 216 independent channels sensor chip had a resolution of $5 \times 10^{-6}$ RIU [35]. Vala et al. fabricated an SPR biosensor with ten sensing channels based on grating coupling with angular interrogation. They obtained a resolution of $6 \times 10^{-7}$ RIU [23] which is close to the detection limit of angular and spectral SPR biosensors.

\subsubsection{Waveguide Couplers}

Moreover, waveguides can be used as couplers to implement SPR biosensors. Wang et al. used a D-type optical fibre as a biosensor by measuring the phase difference between the $\mathrm{p}$ and $\mathrm{s}$ polarisations. The resolution of the device was $2 \times 10^{-6}$ RIU [36]. In Dostálek et al., an optical waveguide had a cladding section covered by a multilayer structure allowing surface plasmons to be excited and to propagate. They input a broadband light source and measured the spectrum at the output of the waveguide to get an SPR dip curve. The resolution of the resulting device was $1.2 \times 10^{-6}$ RIU [37].

Overall, the prism-based coupling allows a lower detection limit to be achieved than with the grating and waveguide approaches. The advantage of waveguide 
and gratings is that they can make much smaller devices and can be cheaper than prism-based SPR biosensors. Another approach to SPR biosensor implementation is to combine two different methods. For example, Bardin et al. combine spectral interrogation to SPR imaging to take advantage of the high sensitivity of spectroscopy in combination with the high throughput allowed by SPR imaging. They illuminate an array of sensing channels in the y axis with a white light source and diffracted the reflecting light using a grating in the $\mathrm{x}$ axis. The array of SPR curves measured on the camera allows multiple and simultaneous analyte sensing in real-time. The resolution of $3.5 \times 10^{-7}$ RIU [38] that they obtained is comparable to spectroscopy SPR biosensors while it is an improvement for SPR imaging devices.

Table 3.2 - Summary of the limit of detection for the different SPR methods

\begin{tabular}{|l|c|}
\hline \multicolumn{1}{|c|}{ SPR method } & Limit of detection \\
\hline Commercial SPR & $10^{-7}$ RIU [20,29] \\
\hline Prism - intensity & $2 \times 10^{-6}$ RIU [30] \\
\hline Prism - angular or wavelength & $2.5 \times 10^{-8}$ RIU [32] \\
\hline Prism - interferometry & $5.5 \times 10^{-8}$ RIU [34] \\
\hline Grating & $6 \times 10^{-7}$ RIU [23] \\
\hline Waveguide & $3.5 \times 10^{-7}$ RIU [38] \\
\hline
\end{tabular}




\section{Chapter 4: General CONCEPT OF SPECTRO-ANGULAR SPR BIOSENSING}

\subsection{System's Description}

The method of interrogation that is used the most for surface plasmon resonance biosensing, as described in Chapter 2, is to measure light intensity with respect to angle or wavelength. The output is a dip curve where the resonant angle or wavelength can be extracted using dip-finding algorithms and one of the most popular algorithms is the centroid method [39,40]. Even though it is very efficient, dip-finding algorithms are still limited by the asymmetry and the noise in the SPR curve. This is why researchers were interested in applying welldeveloped noise reduction and image processing techniques to SPR data. To do so, a two dimensional set of data (an image) is required and to obtain it, SPR dips must be measured with respect to angle and wavelength simultaneously. This biosensor is called a spectro-angular SPR biosensor. Colin Alleyne et al. [14] developed a data processing technique for calculating the refractive index change in spectro-angular SPR biosensor and it will be presented in section 4.2. In the current section, the SPR system required to produce the necessary SPR images will be described.

Figure 4.1 shows the design of the system as elaborated by Alleyne et al. [15]. A collimated broadband source is used at the input and the light goes through a polariser to become TM polarised as it is required for surface plasmon excitation. The cylindrical lens, CL1, focuses the input beam in one dimension to create the range of angles incident on the gold film. The beam then goes through the prism for coupling and reflects on the gold film. The reflected light is collimated with a second and identical cylindrical lens, CL2. Following, the beam reflects on a diffraction grating that diffracts the light in the dimension perpendicular to the 
range of angles; in this dimension the range of wavelengths will be displayed. An output cylindrical lens focuses the diffracted light to a CCD camera which reads a 2D image corresponding to the spectro-angular SPR reflection. The 2D reflectance image shows a dip line corresponding to the absence of reflection due to the surface plasmon excitation. Figure 4.2 gives an example of a 2D SPR image.

Given a change in the refractive index, the resonance conditions will change and the position of the dip line will shift on the angular axis with small changes with respect to wavelength. Figure 4.3 demonstrates the shift in the response due to the change in refractive index from 1.33 RIU (Figure 4.2) to 1.4 RIU (Figure 4.3). The dip line shifts in angle but its shape is modified too; it is wider and more bent towards the right for a greater refractive index. The advantage of this set-up is that fast and real-time measurement can be achieved because there is no time spent waiting on moving parts. The data is acquired in one image all at once. Some of the angular or spectral SPR systems scan the angles or the wavelengths one at the time with a rotation stage or a tunable laser. This takes time and therefore it is not suitable for real-time measurements which commonly involve a data point analysed every five seconds. Measurements are performed in real-time if the analysis is done immediately after each data point is acquired such that the changes occurring in the sample can be monitored, i.e. the data is acquired and analysed faster than the rate at which the changes are happening. The analysis speed therefore varies for different types of measurements. For biosensing, a data point every few seconds, such as one per five seconds, is appropriate. In the spectro-angular biosensor presented here, the data is acquired quickly and is processed in real-time allowing the device to monitor the refractive index of the sample. 


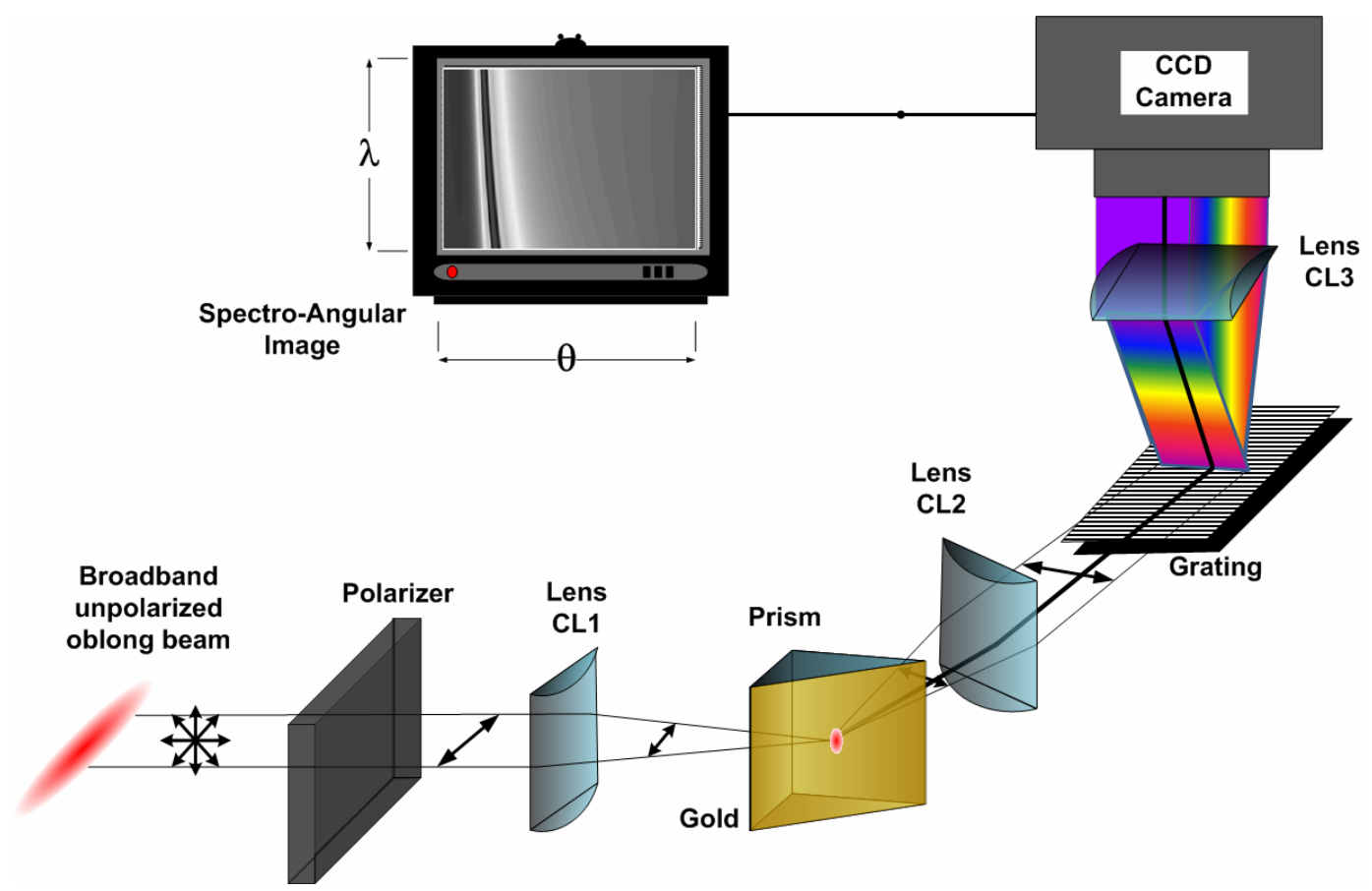

Figure 4.1 - Design of the spectro-angular biosensor [41]

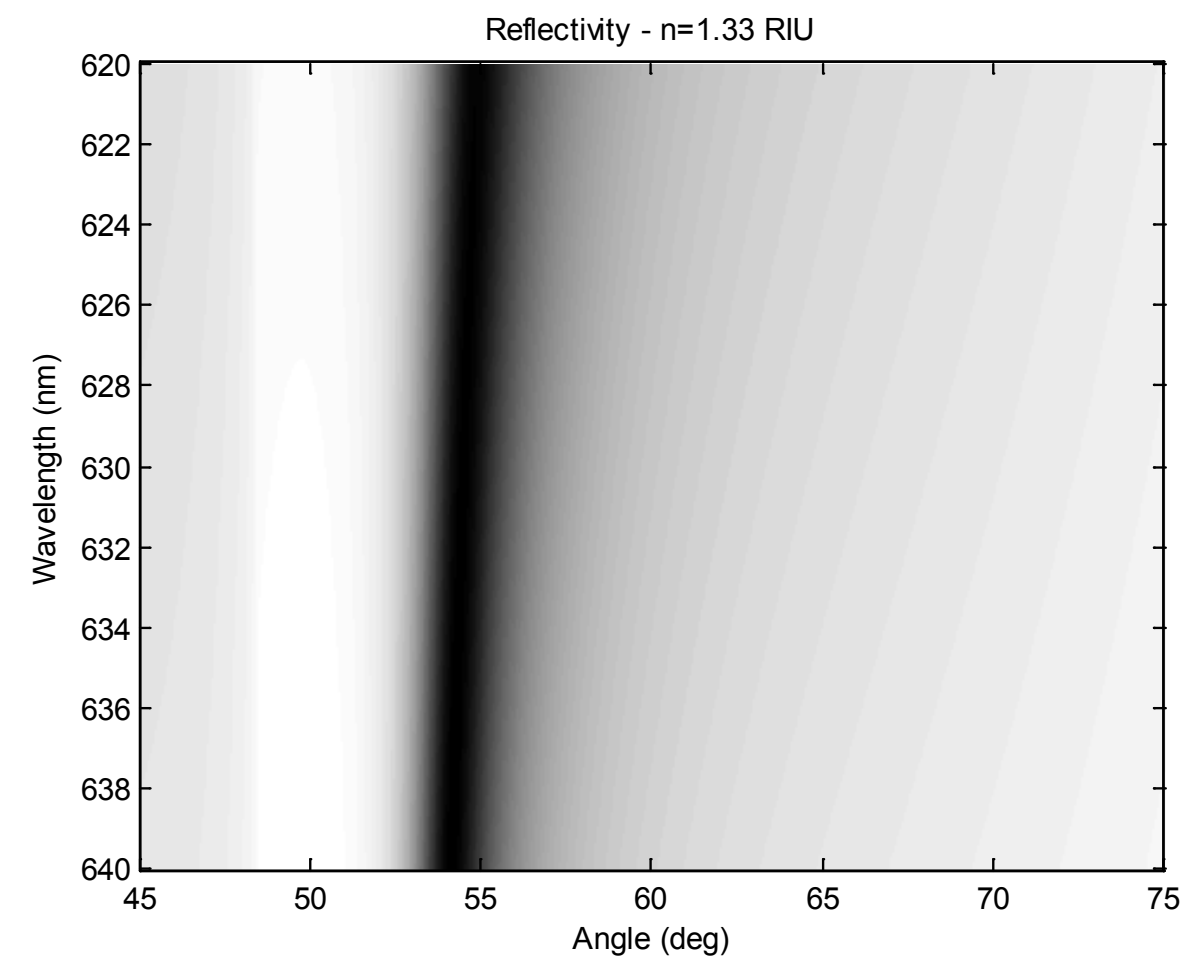

Figure 4.2 - Spectro-angular SPR response for a refractive index of 1.33 RIU 


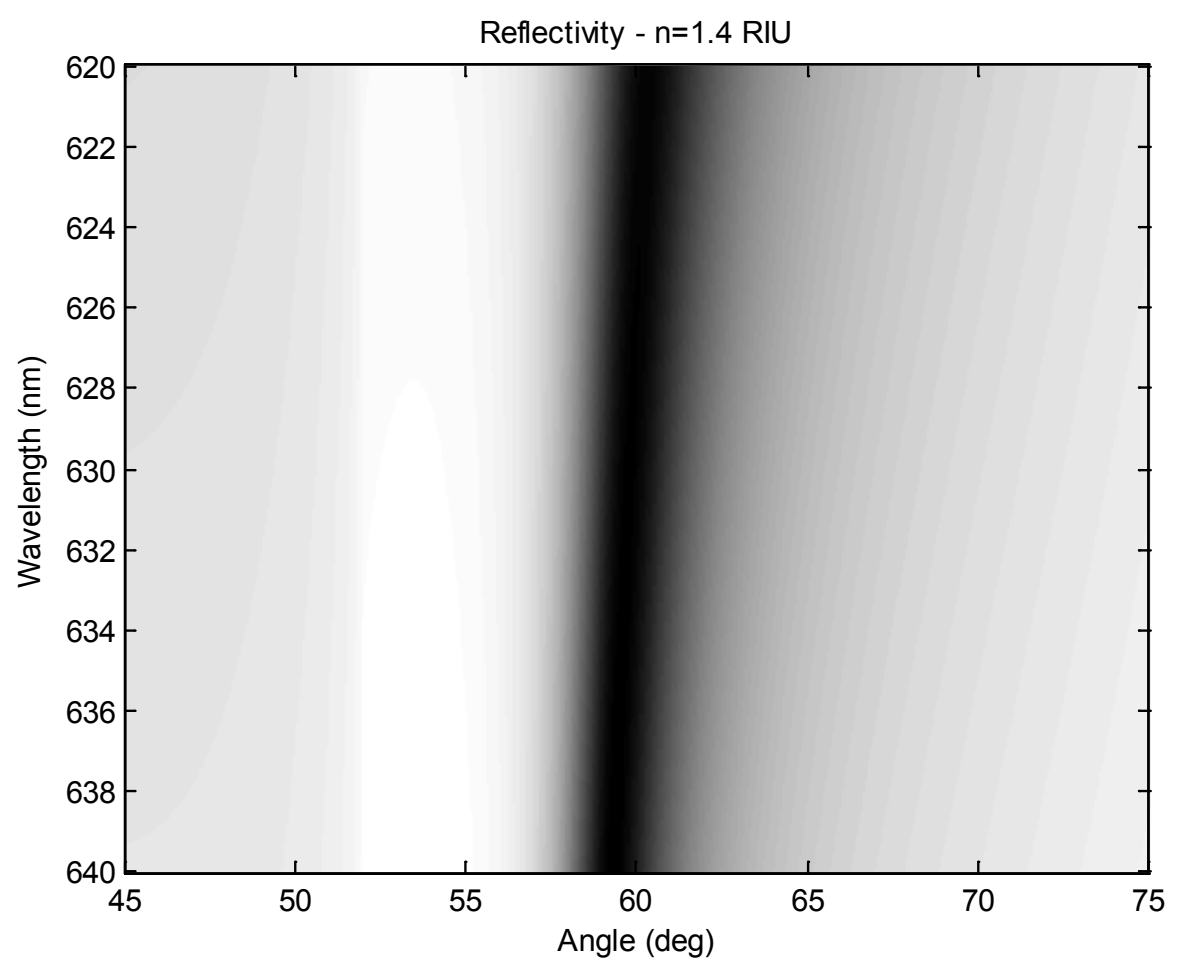

Figure 4.3 - Spectro-angular SPR response for a refractive index of 1.4 RIU

\subsection{The Double Projection Method}

With SPR biosensing, it is very important to use a good data processing technique. The efficiency of the system will highly depend on it. As mentioned previously, most SPR sensors use a dip-finding algorithm such as the centroid method. In the case of spectro-angular reflectance measurements, image processing techniques can be used to analyse the data. A new technique was developed by Alleyne et al. [14]: the double projection method (DPM). This method consists in simulating a set of SPR images corresponding to multiple refractive indices across the dynamic range of the biosensor and comparing the experimental images with the simulated images to estimate the unknown refractive indices. The absolute value of the refractive index of a sample depends on too many variables to be measured exactly but the change in refractive index can be precisely measured with this technique. 
The first step consists in simulating the reflection images of known refractive indices that will form the training set. Given the parameters of the experimental set-up, the dielectric constant and thickness of the metal, the coupling method, the required range of wavelengths and angles, the resolution of the camera, as well as the refractive indices of interest, the output images can easily be simulated in Matlab with the SPR reflection equations (equations 2.5 to 2.7). A basis set is then built using singular value decomposition (SVD). SVD is the factorization of a matrix $m \times n$ into three matrices, $\mathbf{M}=\mathbf{U} \boldsymbol{\Sigma} \mathbf{V}^{\mathbf{T}}$, where $\mathbf{U}$ is a $m x m$ orthogonal matrix, $\boldsymbol{\Sigma}$ is a $m x n$ diagonal matrix containing the singular values of $\mathbf{M}$ and $\mathbf{V}^{\mathbf{T}}$ is a $n x n$ orthogonal matrix. The matrix of interest for the DPM is the matrix $\mathbf{U}$ in which the columns are the eigenvectors of $\mathbf{M} \mathbf{M}^{\mathbf{T}}$.

The training set, $\mathbf{a}_{\mathbf{i}}$, is made of a set of normalised vectors where each vector corresponds to an SPR image of a given refractive index. In other words, each column of $\mathbf{a}_{\mathbf{i}}$ is a 2D SPR image rearranged into a vector. Performing the SVD on $\mathbf{a}_{\mathbf{i}}$ as the matrix $\mathbf{M}$ gives the basis $\mathbf{b}_{\mathbf{j}}$ which is formed by the columns of the matrix $\mathbf{U}$. The next step consists in performing the first projection; the matrix $\mathbf{a}_{\mathbf{i}}$ containing the training set is projected on the basis $\mathbf{b}_{\mathbf{j}}$ to give the weight matrix $\mathbf{A}$. The projection is done with the inner product operator, $\mathbf{A}=\mathbf{a}_{\mathbf{i}} \cdot \mathbf{b}_{\mathbf{j}}$. $\mathbf{A}$ and $\mathbf{b}_{\mathbf{j}}$ are kept in memory and will be used to estimate the refractive indices of experimental images in real-time.

With the basis in memory, the refractive index estimation is done as follows. Every image of unknown refractive index is rearranged into a column vector $\mathbf{v}$, and is projected against $\mathbf{b}_{\mathbf{j}}$ to create the weight vector $\mathbf{w}_{\mathbf{j}}$. The weight vector $\mathbf{w}_{\mathbf{j}}$ is then projected against each row in the weight matrix $\mathbf{A}$ to give the vector $\mathbf{s}, \mathbf{s}=\mathbf{A} \cdot$ $\mathbf{w}_{\mathbf{j}}$. The vector $\mathbf{s}$ plotted with respect to the refractive indices of the training set shows a curve where the maximum point corresponds to the best match between the unknown image and the images of the training set. After this second projection (hence the name of the method, the double projection method), the maximum point is extracted from the vector $\mathbf{s}$ using interpolation and the 
corresponding abscissa will give the estimated refractive index with high precision. Figure 4.4 shows a summary of this method.

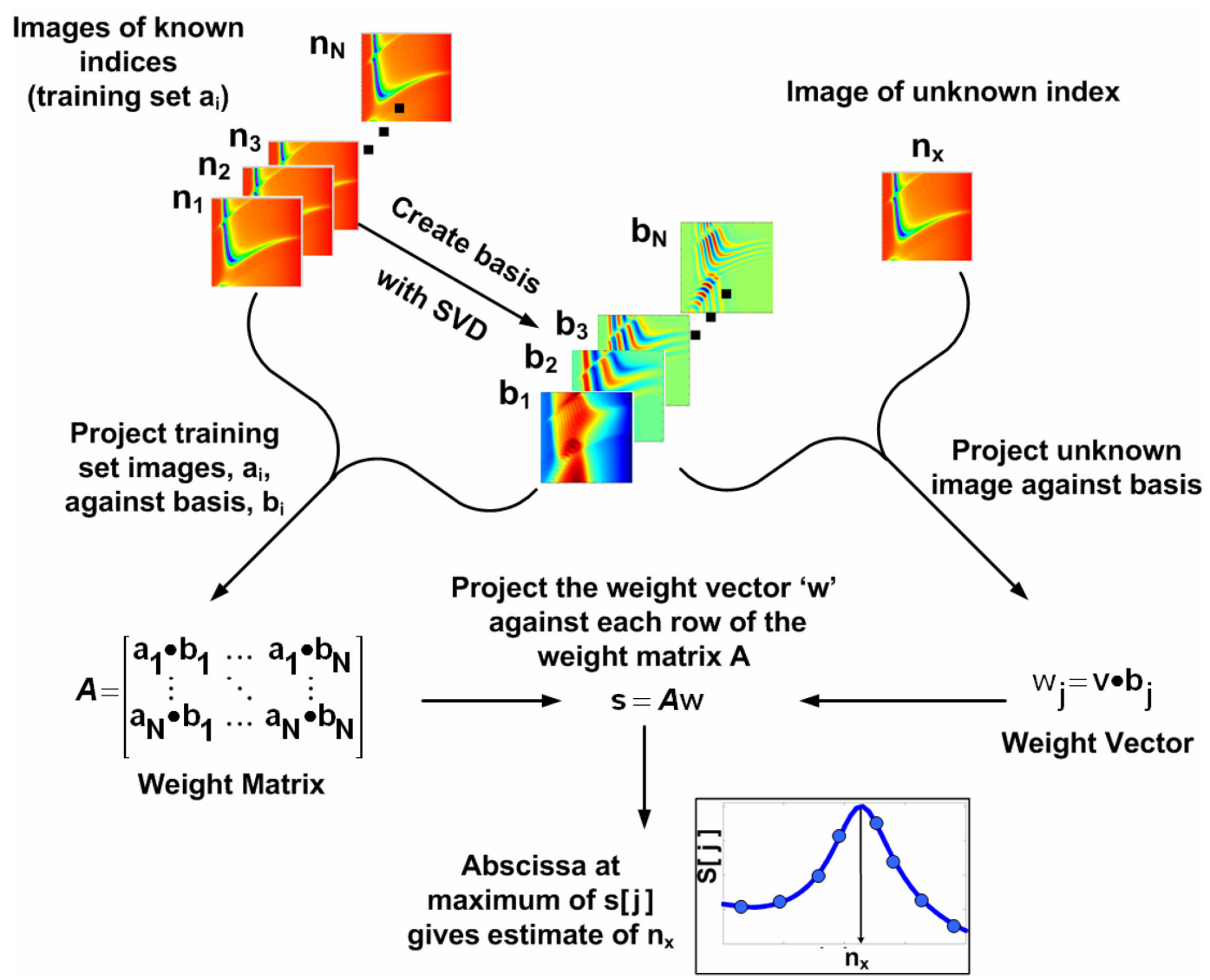

Figure 4.4 - Summary of the Double Projection Method for refractive index estimation [14]

Tracking the estimated refractive index in real-time will allow the user to obtain an accurate measurement of the change in refractive index. In theory, the DPM can calculate the change in the refractive index of a sample with a resolution of $5 \times 10^{-9}$ RIU [15] for noise-free data. Experimental data obtained from a real-time monitoring of the refractive index were analysed both with the DPM and a weighted centroid dip finding algorithm and the signal-to-noise ratio (SNR) was $42.4 \mathrm{~dB}$ for the DPM and $15 \mathrm{~dB}$ for the centroid method [14] which shows the DPM offers a better precision in the results. Another advantage of this method 
over the dip finding algorithm is that the sensitivity with angular or spectral SPR is not actually linear. In other words, the shift in angle of incidence or wavelength around 1.3 RIU is not exactly the same as the shift around 1.4 RIU; in fact it is smaller at 1.3 RIU. Therefore this is a parameter that has to be taken into account when using the SPR system as a biosensor. However, with the DPM since the method involves a comparison between the experimental results and a precomputed basis, the non-linearity of the shift is automatically considered.

\subsection{Noise Reduction: Temperature Control, Reference Channel and Normalisation}

One of the main challenges with SPR biosensor is to deal with the temperature change. The light beam incident on the gold surface and the plasmon propagating on the metal-dielectric interface produce a rise in the temperature of the sample through time. The refractive indices of liquids are dependent on the temperature since the temperature changes the density of liquids which is linked to the refractive index. As the temperature increases, the density of liquids decreases and therefore the refractive index will decrease as well. For example, water has a refractive index of 1.3321 [42] at $630 \mathrm{~nm}$ at $21.5^{\circ} \mathrm{C}$. As the temperature increases, the refractive index of water at $630 \mathrm{~nm}$ decreases by $94.3 \times 10^{-6} \mathrm{RIU} /{ }^{\circ} \mathrm{C}$ [42]. This makes a big difference in the refractive index change measurement as the objective is to design a system with a resolution of $10^{-7}$ RIU or better.

The first technique applied to limit the temperature increase is to use a thermoelectric cooler (TEC). A TEC is a device made of two plates that experience a difference in temperature when a voltage is applied across the plates. By putting the cold face adjacent to the sample, the TEC cools the sample to a given temperature. The temperature of the sample will in fact fluctuate slightly around the set temperature within the precision of the TEC controller. The TEC controller from Wavelength Electronics used in this work has a linear temperature stability of $0.003^{\circ} \mathrm{C}$. The temperature control helps a lot the refractive index 
change measurement by ceasing the constant increase but it is not perfect and the remaining fluctuations must be dealt with.

The best way to reduce this fluctuation noise as well as the other sources of noise, such as the power fluctuations in the light source and the background light, is to introduce a reference channel which shares common parameters with the signal channel. Both channels must be driven by the same light source, must traverse identical optical components, must have a common temperature control and their output signal must be measured by identical cameras at the same time. The only difference is the refractive indices of the sample. In the reference channel, the buffer alone is constantly flowing through the reference chamber while the refractive index change measurement is happening in the signal channel. Both channels will experience the same temperature fluctuations as well as various other sources of noise so the estimated index of the reference channel can be subtracted from the estimated index of the signal channel to suppress the effects of the different noise sources. After the subtraction, only the change in refractive index going on in the signal chamber will show up in the results since the fluctuations should be greatly reduced.

To set-up a useful reference channel, the light source is split in two beams. This allows both channels to share the same power fluctuations. Also, the two sensing regions must be as close as possible to be cooled down equally by the same TEC. Additionally, the most important aspect for the good functioning of the reference channel is to acquire the data from each channel simultaneously. There are two ways of doing so: by acquiring the data from both channels on two identical and synchronised cameras or on the same camera. Both methods will be discussed later in chapters 5 and 6 respectively.

Finally, before estimating the refractive index, the data of each channel is normalised to remove the undesired effects created by the optical system. Different spatial patterns show up, such as interference patterns, on the 
experimental images because of the optical components. In order to clear up the data for a better processing, the output image is normalised. The normalisation is done with an image taken with air in the sample chamber, before the sample is introduced in the chamber, to remove the light patterns. With the parameters of the biosensor chosen such that a surface plasmon is excited with water as the sample, when the sensing chamber is filled with air, no surface plasmons are excited. The light totally reflects but since it goes through the same optical path, any noise resulting from the optical components will be removed by normalising the SPR images with the air image. The advantage is also that it will reduce the effect of the Gaussian distribution of the spectrum. The simulation performed for the training set assumes a flat spectrum, i.e. the same power for all wavelengths. In reality, the spectrum is Gaussian and the data normalisation will increase the match between the experimental image and the training set images. 


\section{Chapter 5: NEW SPR BIOSENSOR}

\subsection{Motivation and Simulations}

Although the theoretical resolution of the spectro-angular SPR biosensor with the Double Projection Method is $5 \times 10^{-9}$ RIU, a resolution of $1.5 \times 10^{-6}$ RIU was achieved with the experimental set-up[14,15]. This is mainly due to the specifications of the cameras and the noise (camera noise and temperature fluctuations). As shown by Alleyne et al. [14], the spatial resolution of the cameras and the precision in the intensity levels (number of bits used in the analog-to-digital converter (ADC) of the cameras) make a great difference in the refractive index estimation error. Figure 5.1 shows those results; a larger spatial resolution produces a smaller estimation error and as the number of bits used in the ADC increases the root-mean-square (RMS) error reduces significantly. This is where the motivation for the research presented in this thesis lies. The cameras used in the previous set-up had a resolution of 480x640 and used a 10-bit ADC. The objective of the current project is to reduce the detection limit of the spectroangular SPR biosensor by upgrading the cameras parameters.

The first thing to consider is the wavelength at which the system operates. The previous system was operating at $850 \mathrm{~nm}$ which was an appropriate wavelength for SPR measurements because the SPR curve at $850 \mathrm{~nm}$ has a much sharper dip and a deeper sensing region in the sample than at shorter wavelengths. However, this choice of wavelength involves the use of near-infrared cameras. The cameras sensitive for near-infrared wavelengths do not offer a spatial resolution and an intensity level resolution as great as CCD sensors in the visible spectrum and are more expensive. Therefore, cameras sensitive in the visible spectrum will be chosen for the new system. The change of wavelength from $850 \mathrm{~nm}$ to the visible should not decrease the performance of the biosensor. Since the data analysis is not dependent on a dip finding algorithm, the refractive index estimation is 
virtually unaffected by the change in wavelength but improved through the increase in spatial resolution data precision as it will be shown with the following simulations.

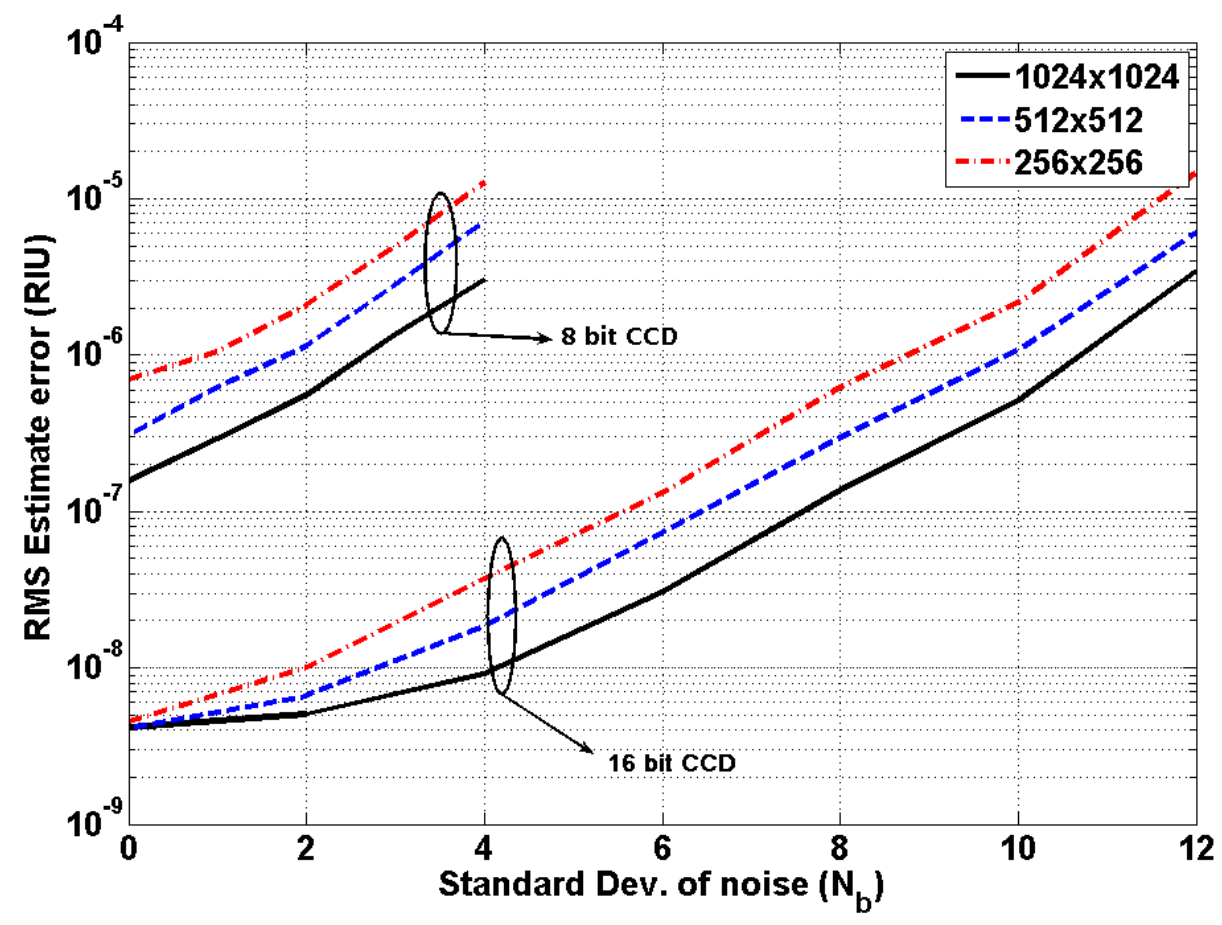

Figure 5.1 - RMS Error for the estimation of a refractive index change of $10^{-6}$ and a training set with intervals of 0.002 RIU for different camera resolutions $(256 \times 256,512 \times 512$ and $1024 \times 1024)$ and for different ADC levels (8-bit and 16-bit). The noise added for each pixel (x-axis) is Gaussian with a standard deviation of $2^{\mathrm{Nb}-1} / 2^{\mathrm{n}}-1$ where $N_{b}$ is the number of bit of noise and $n$ is the number of bit used in the ADC.

A wavelength of $630 \mathrm{~nm}$ was chosen for the new system. Figure 5.2 and Figure 5.3 show both SPR dips at $850 \mathrm{~nm}$ and $630 \mathrm{~nm}$ with the same range of angles and the same range width of wavelengths. It demonstrates how sharper the SPR dip is at $850 \mathrm{~nm}$ than at $630 \mathrm{~nm}$. The sensing regions in water in both cases are $394 \mathrm{~nm}$ and $178 \mathrm{~nm}$ respectively and the propagation lengths of the plasmon on the interface are $24 \mu \mathrm{m}$ and $3 \mu \mathrm{m}$ respectively. The $630 \mathrm{~nm}$ wavelength chosen for the 
new system is well in the range of detection of cameras operating in the visible spectrum and has a good SPR dip shape. The sensitivity of those cameras is better at shorter wavelengths, towards blue, than at red wavelengths but short wavelengths have a shallow SPR dip which could decrease the performance of the biosensor. The dip is very broad for wavelengths around $500 \mathrm{~nm}$ and the extinction ratio is small compared to longer wavelengths as shown on Figure 5.4. This would make it hard to extract a sufficient dip for analysis. However, the assumption was made that regardless of the dip shape, it was possible to estimate the refractive index with the DPM which could mean that it would still be possible to use $500 \mathrm{~nm}$. The propagation length and the width of the sensing region have to be considered as well. At $500 \mathrm{~nm}$, the propagation length is 0.009 $\mu \mathrm{m}$, which is 300 times smaller than at $630 \mathrm{~nm}$, and the sensing region is $87 \mathrm{~nm}$, which is half the size of $630 \mathrm{~nm}$. The small propagation length and sensing region suggest that the SPR sensitivity to the change in the refractive index would be poor. Therefore at $630 \mathrm{~nm}$ we get the best case, a good resolution and precision in the cameras with enough sensitivity and a good SPR dip. 


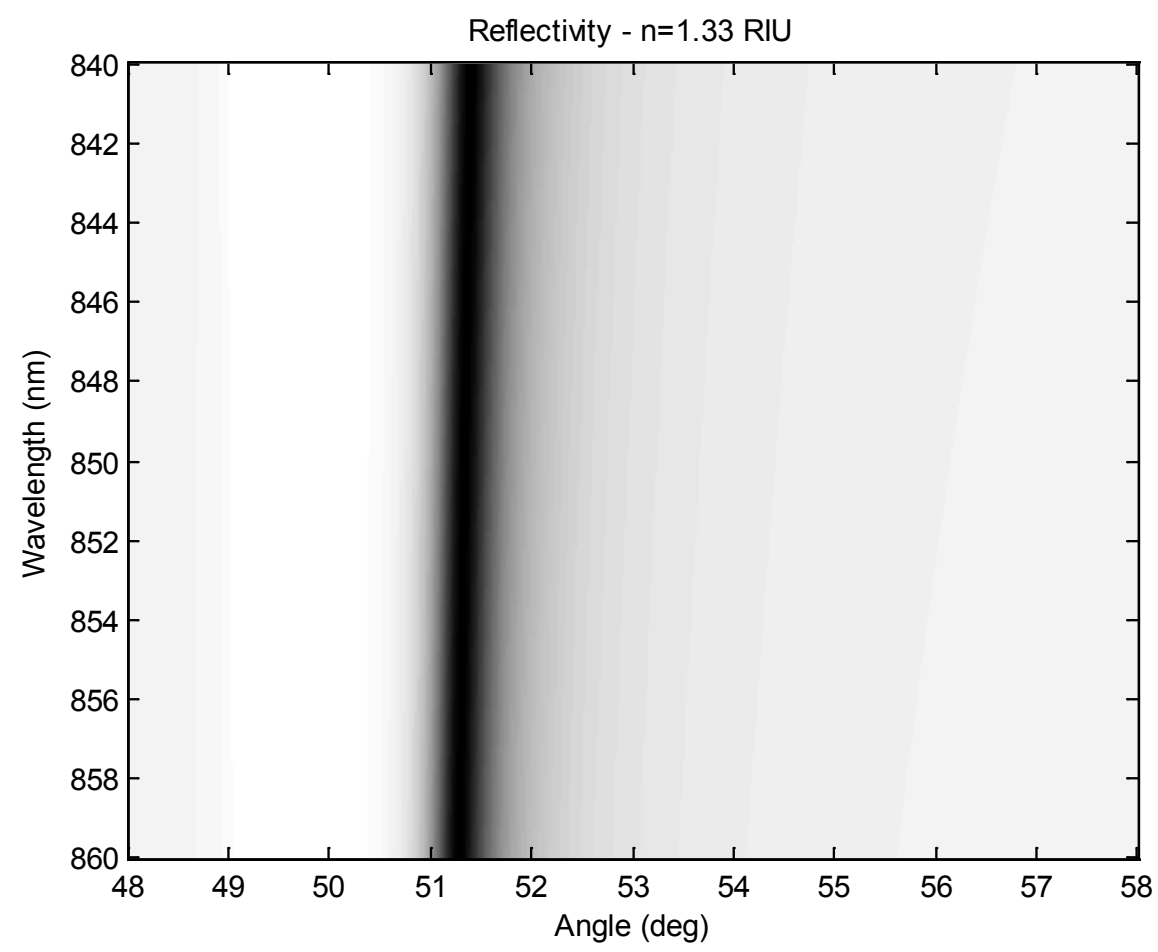

Figure 5.2 - Spectro-angular SPR response centered at $850 \mathrm{~nm}$ for a refractive index of $1.33 \mathrm{RIU}$

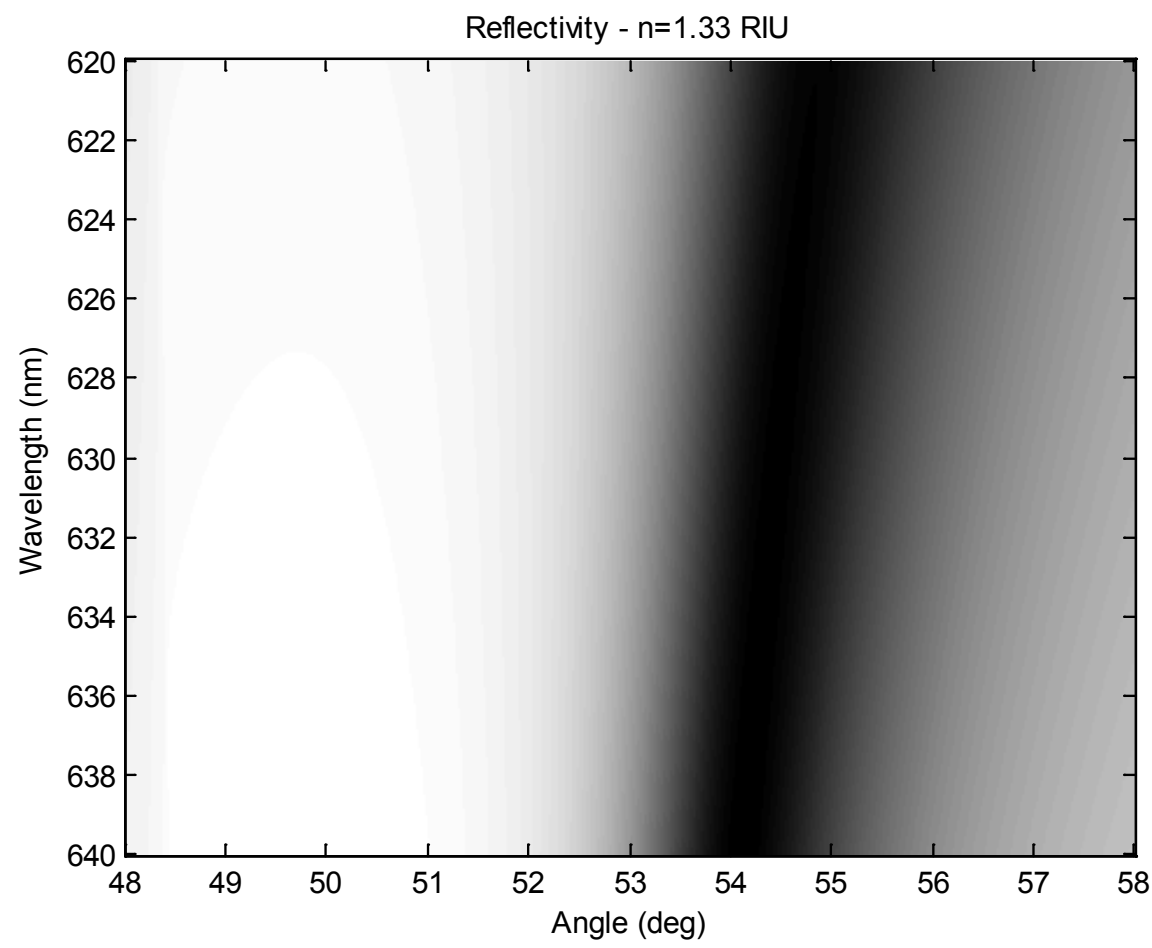

Figure 5.3 - Spectro-angular SPR response centered at $630 \mathrm{~nm}$ for a refractive index of 1.33 RIU 


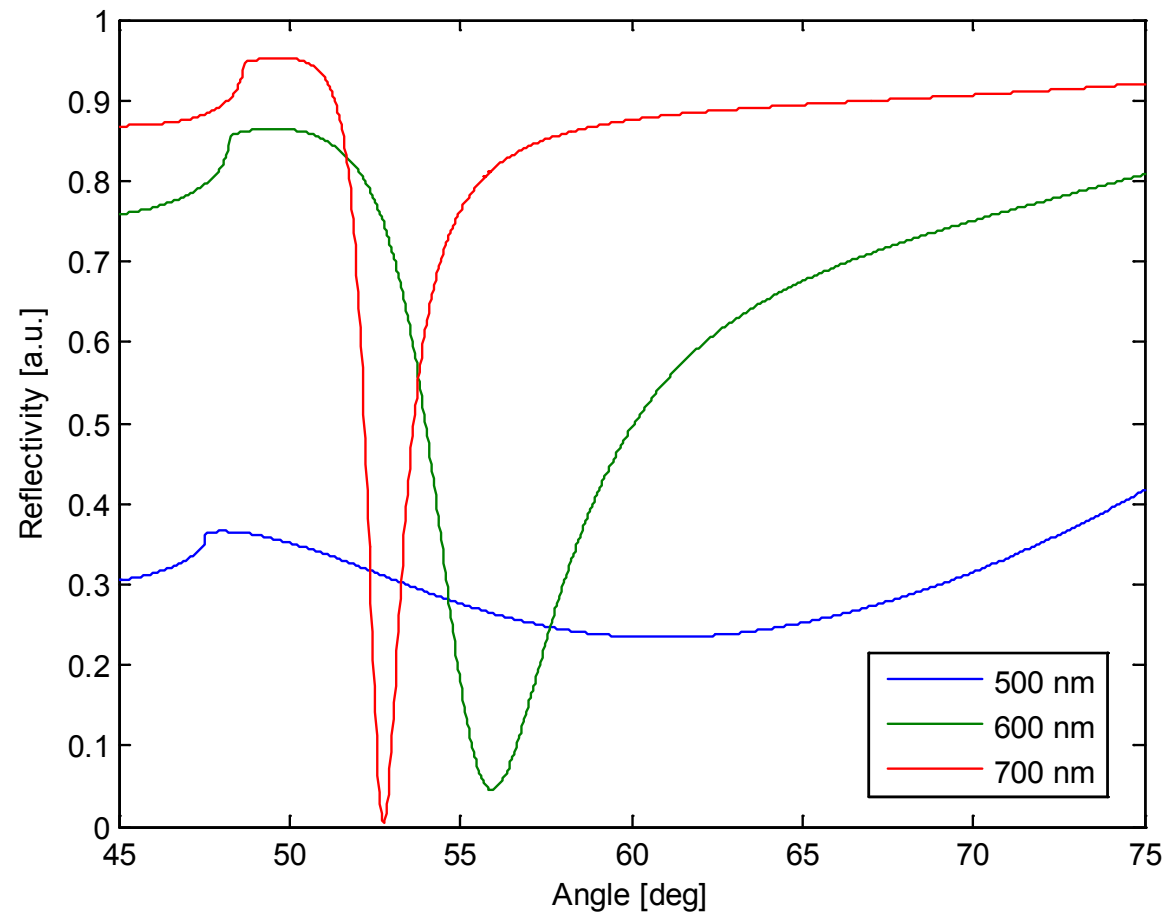

Figure 5.4 - SPR dip at three different wavelengths across the visible spectrum $(500 \mathrm{~nm}, 600 \mathrm{~nm}$ and $700 \mathrm{~nm})$. The refractive index of the dielectric is $1.33 \mathrm{RIU}$.

To validate the change of wavelength, the RMS error in estimating refractive index changes is calculated for both $850 \mathrm{~nm}$ and $630 \mathrm{~nm}$ using Matlab. In the first simulation, the same parameters for both wavelengths are used: a spatial resolution of $1024 \times 1024$, a range of angles of $10^{\circ}$ and a range of wavelengths of $20 \mathrm{~nm}$. In both cases the training set consists of 51 images representing refractive index from 1.30 to $1.35 \mathrm{RIU}$ (equation 5.1), therefore with an interval of 0.001 RIU.

$$
n_{k}^{\text {train }}=\left\{\begin{array}{c}
1.300 \\
1.301 \\
\vdots \\
1.350
\end{array}\right.
$$


The test images are chosen to represent the worst-case scenario. The worst-case scenario is when the test images correspond to refractive indices in between the refractive indices of the training set. Equation 5.2 shows the main test set, $\mathrm{n}^{\mathrm{t}}$. Three other test sets of images, $\mathrm{n}^{\mathrm{tt}}$, are create to estimate different changes in refractive index, $\Delta=10^{-6}, 10^{-7}$ and $10^{-8}$, with respect to the main test set. Equation 5.3 gives an example of $\mathrm{n}^{\mathrm{tt}}$ with $\Delta=10^{-6}$.

$$
\begin{gathered}
n_{k}^{t}=\left\{\begin{array}{c}
1.3005 \\
1.3015 \\
\vdots \\
1.3495
\end{array}\right. \\
n_{k}^{t t}=\left\{\begin{array}{c}
1.300501 \\
1.301501 \\
\vdots \\
1.349501
\end{array}\right.
\end{gathered}
$$

The refractive index change is estimated for each image in the sets and the estimation error is calculated. The RMS error is then used to compare the performance at both wavelengths.

$$
\begin{gathered}
\delta_{k}=\left|\left(\hat{n}_{k}^{t t}-\hat{n}_{k}^{t}\right)-\Delta\right| \\
\delta_{R M S}=\sqrt{\sum_{k} \frac{\delta_{k}^{2}}{N}}=\sqrt{\sum_{k} \frac{\left|\left(\hat{n}_{k}^{t t}-\hat{n}_{k}^{t}\right)-\Delta\right|^{2}}{N}}
\end{gathered}
$$

$\delta_{\mathrm{k}}$ is the $\mathrm{k}^{\mathrm{th}}$ estimation error, $\delta_{\mathrm{RMS}}$ is the RMS error, $\hat{n}_{k}$ is the $\mathrm{k}^{\text {th }}$ estimated refractive index and $\mathrm{N}$ is the number of images in the test set which in this case is 50. Table 5.1 shows the simulation results. 
Table 5.1 - RMS estimation error for different refractive index changes using the same cameras

\begin{tabular}{|c|c|c|c|c|c|c|}
\hline \multirow[t]{2}{*}{$\begin{array}{l}\text { RI } \\
\text { change } \\
(\Delta)\end{array}$} & \multirow{2}{*}{\multicolumn{2}{|c|}{$\begin{array}{l}\text { Error } \\
\text { (no noise) } \\
630 \mathrm{~nm} \quad 850 \mathrm{~nm}\end{array}$}} & \multirow{2}{*}{\multicolumn{2}{|c|}{$\begin{array}{l}\text { RMS Estimation } \\
\text { Error } \\
\text { (4-bit noise) } \\
630 \mathrm{~nm} \quad 850 \mathrm{~nm}\end{array}$}} & \multirow{2}{*}{\multicolumn{2}{|c|}{$\begin{array}{l}\text { RMS Estimation } \\
\text { Error } \\
\text { (5-bit noise) } \\
630 \mathrm{~nm} \quad 850 \mathrm{~nm}\end{array}$}} \\
\hline & & & & & & \\
\hline $10^{-6}$ & $1.414 \mathrm{e}-9$ & $7.211 \mathrm{e}-9$ & $8.718 \mathrm{e}-9$ & $9.798 \mathrm{e}-9$ & $2.248 \mathrm{e}-8$ & $1.363 \mathrm{e}-8$ \\
\hline $10^{-7}$ & $1.414 \mathrm{e}-9$ & $2.449 \mathrm{e}-9$ & $8.000 \mathrm{e}-9$ & $7.211 \mathrm{e}-9$ & $2.067 \mathrm{e}-8$ & $1.240 \mathrm{e}-8$ \\
\hline $10^{-8}$ & $7.094 \mathrm{e}-17$ & $7.094 \mathrm{e}-17$ & $9.695 e-9$ & $6.928 \mathrm{e}-9$ & & $1.172 \mathrm{e}-8$ \\
\hline
\end{tabular}

Any results in the order of $10^{-16}$ or $10^{-17}$ should be considered to be zero as it is due to the finite numerical precision of Matlab. The results shown with "4-bit noise" and "5-bit noise" are the RMS estimation error when the test images were modified to add Gaussian noise with a standard deviation of $2^{\mathrm{Nb}-1} / 2^{\mathrm{n}}-1$ where $\mathrm{N}_{\mathrm{b}}$ is the number of bits of noise ( 4 and 5 bits in this case) and $n$ is the number of bit in the ADC (16-bit). The level of noise was chosen such that the simulation results under the experimental conditions would be close to the detection limit found with the experimental system, $1.5 \times 10^{-6}$ RIU. In this case, the level of noise required is 5 bits. The results presented in Table 5.1 demonstrate that changing the wavelength of the source will not significantly change the precision in the refractive index estimation with respect to the data analysis. In other words, the dip shape at $630 \mathrm{~nm}$ does not affect the estimation with the DPM. However, the same simulations were performed at $510 \mathrm{~nm}$ and the results show a drop by an order of magnitude in the noisy case which confirms that $630 \mathrm{~nm}$ is a better option.

Since the objective is the improvement of the resolution of the experimental sensor, better cameras in terms of spatial resolution and intensity level resolution have to be used. The second simulation to compare between $630 \mathrm{~nm}$ and $850 \mathrm{~nm}$ is to use the same angular range and the same range of wavelengths as before but 
different camera parameters. In the $850 \mathrm{~nm}$ case, the camera has a resolution of 480x640 and uses a 10-bit ADC. In the $630 \mathrm{~nm}$ case, the camera has a resolution of 1024x1024 and uses a 14-bit ADC. The RMS errors are shown in Table 5.2 for noise-free data and with 4-bit of noise. The results demonstrate that the detection limit should improve upon using a camera and a light source operating in the visible spectrum. Considering 4 bits of noise, the theoretical resolution is improved 14-fold for the new parameters and for 5 bits of noise it is improved by a factor of about 16. This suggests that it will be possible to detect changes better than $10^{-7}$ RIU.

Table 5.2 - RMS estimation error for different refractive index changes using different cameras

\begin{tabular}{|c|c|c|c|c|c|c|}
\hline \multirow[t]{2}{*}{$\begin{array}{l}\text { RI } \\
\text { change } \\
(\Delta)\end{array}$} & \multirow{2}{*}{\multicolumn{2}{|c|}{ 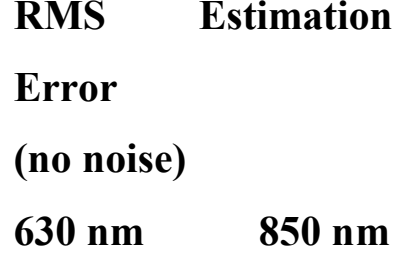 }} & \multirow{2}{*}{\multicolumn{2}{|c|}{$\begin{array}{l}\text { RMS Estimation } \\
\text { Error } \\
\text { (4-bit noise) } \\
630 \mathrm{~nm} \quad 850 \mathrm{~nm}\end{array}$}} & \multirow{2}{*}{\multicolumn{2}{|c|}{$\begin{array}{l}\text { RMS Estimation } \\
\text { Error } \\
\text { (5-bit noise) } \\
630 \mathrm{~nm} \quad 850 \mathrm{~nm}\end{array}$}} \\
\hline & & & & & & \\
\hline $10^{-6}$ & $1.147 \mathrm{e}-16$ & $3.397 \mathrm{e}-8$ & $3.924 \mathrm{e}-8$ & $5.804 \mathrm{e}-7$ & $8.290 \mathrm{e}-8$ & $1.352 \mathrm{e}-6$ \\
\hline $10^{-7}$ & $1.549 \mathrm{e}-16$ & $1.273 \mathrm{e}-8$ & $4.264 \mathrm{e}-8$ & $5.623 e-7$ & $8.356 \mathrm{e}-8$ & $1.304 \mathrm{e}-6$ \\
\hline $10^{-8}$ & $7.094 \mathrm{e}-17$ & $4.810 \mathrm{e}-9$ & $4.308 \mathrm{e}-8$ & $6.878 \mathrm{e}-7$ & $8.890 \mathrm{e}-8$ & $1.331 \mathrm{e}-6$ \\
\hline
\end{tabular}

\subsection{Design and Components}

\subsubsection{Main Components}

The light source chosen for the current project is a fibre-coupled light emitting diode (LED) centered at $630 \mathrm{~nm}$ with a spectrum of $17.9 \mathrm{~nm}$ and an output power of $5.2 \mathrm{~mW}$. Table 5.3 and Figure 5.5 show the specifications of the light source purchased from Doric lenses. A broadband source was required for the implementation of the spectro-angular biosensor and therefore lasers could not be 
used as they are monochrome. An interesting option for a broadband light source was a super luminescent diode (SLD), as it was used previously, but SLD are not commercially available in the visible spectrum. Therefore, the LED was the best solution. It is a broadband light source offered at various choices of center wavelengths and can achieve high output power. The output power is an important parameter to consider since some losses are expected in the free-space set-up and the power must be high enough to produce a sufficient signal on the cameras.

The only drawback in choosing an LED as the light source is the necessary use of a multimode optical fibre. The large emitting area of LED requires to be coupled into a large-core optical fibre to minimise the power loss. Even though the system is built with free-space optical components, the LED is coupled in an optical fibre to use a fibre coupler to split the light for both signal and reference channels instead of a free-space beam splitter. Unlike the previous design, the fibre coupler was favoured over the beam splitter in this case to reduce the loss in off reflections and decrease the difficulty in alignment imposed by a free-space beam splitter. A $3 \mathrm{~dB}$ fibre coupler was purchased from FONT Canada with fibre specifications matching those of the input fibre to minimise the loss. In order to benefit from the high power delivery of the LED, the light must be coupled into a large-core fibre. Single-mode fibres can be used to couple the light from an LED but the loss is too great and the output power would not be large enough for this application. The challenge with free-space optics and a large-core fibre is the difficulty to collimate the light. The source must be treated as an extended source due to its multimode nature and therefore it is not possible to perfectly collimate the light. It will be shown later in this section how this challenge was dealt with. 
Table 5.3 - Specifications of the fibre-coupled LED from Doric lenses

\begin{tabular}{|l|l|}
\hline Peak wavelength & $630 \mathrm{~nm}$ \\
\hline Spectral bandwidth FWHM & $17.9 \mathrm{~nm}$ \\
\hline Output power & $5.2 \mathrm{~mW}$ \\
\hline Core diameter of the fibre & $400 \mu \mathrm{m}$ \\
\hline Numerical aperture of the fibre & 0.39 \\
\hline
\end{tabular}

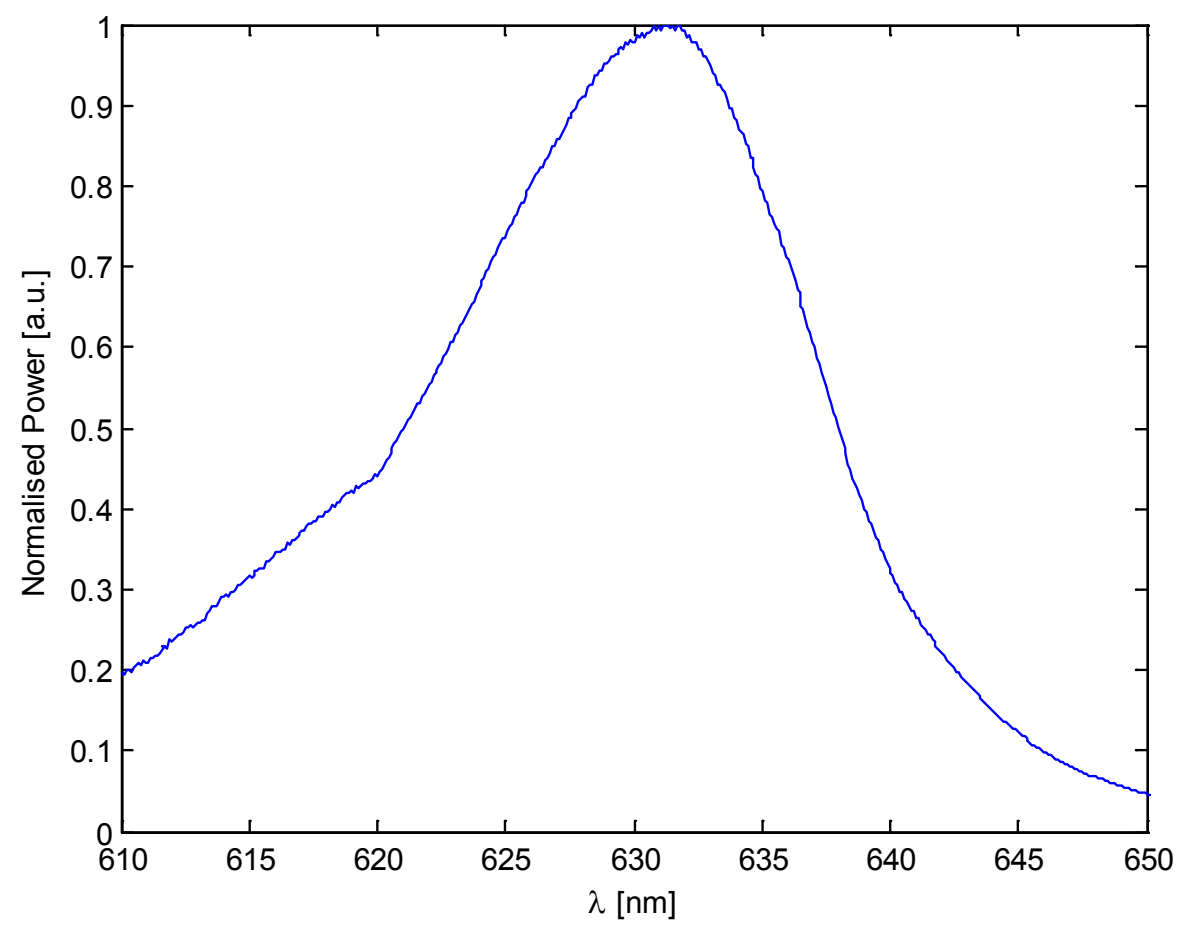

Figure 5.5 - Output spectrum of the fibre-coupled LED measured by an optical spectrum analyser

CCD cameras were chosen to improve as much as possible the performance of the biosensor by upgrading the main features, the spatial resolution (the number of pixels) and the number of bits in the analog to digital converter (ADC). Table 5.4 shows the comparison between the previously used cameras, Micronviewer 7290A from Electrophysics, and the new ones, Pike F-421B from Allied Vision 
Technology. Additionally from having more pixels and more precision, the new cameras are much smaller, are lighter and have smaller pixels. The weight and size are important characteristics when it comes to fabricate a more compact and ultimately portable system.

The other main difference between the two cameras is the image acquisition. Previously, the cameras were connected to an image acquisition card and a frame rate of 30 frames per second was achievable. However, even if the cameras were synchronised, it was not possible to acquire the two corresponding images. The acquisition was done on one channel at a time discarding the image from the other channel. With the AVT cameras, the interface with the computer is through FireWire and the on-board buffer allows the acquisition of all the images. Therefore the two corresponding images are obtained and this increases the efficiency of the reference channel. Additionally, the AVT cameras offer on-board averaging which is a valuable feature as it will be explained in section 5.3. The Pike F-421B cameras are equipped with the Kodak sensor KAI-04022. Table 5.5 shows the specifications of the sensors for one pixel.

Table 5.4 - Comparison between the specifications of the Micronviewer and the Pike cameras

\begin{tabular}{|l|l|l|}
\hline Specifications & Micronviewer 7290A & Pike F-421B \\
\hline Resolution & $640 \times 480$ & $2048 \times 2048$ \\
\hline ADC & $10 \mathrm{bit}$ & $14 \mathrm{bit}$ \\
\hline Pixel size & $18 \times 18 \mu \mathrm{m}$ & $7.4 \times 7.4 \mu \mathrm{m}$ \\
\hline Spectral range & $0.4-1.9 \mu \mathrm{m}$ & $0.35-1 \mu \mathrm{m}$ \\
\hline Body Dimension & $22 \times 10 \times 7 \mathrm{~cm}$ & $9.68 \times 4.4 \times 4.4 \mathrm{~cm}$ \\
\hline Weight & $1.13 \mathrm{~kg}$ & $0.250 \mathrm{~kg}$ \\
\hline
\end{tabular}


Table 5.5 - Parameters of the AVT Pike camera and Kodak Sensor

\begin{tabular}{|l|l|}
\hline Parameters & F-421B / KAI-04022 \\
\hline Quantum efficiency, $\eta$ & 0.34 (at $630 \mathrm{~nm})$ \\
\hline Full well capacity, FW & $40000 \mathrm{e}-$ \\
\hline Read noise, $\mathrm{n}_{\mathrm{R}}{ }^{*}$ & $9 \mathrm{e}-\mathrm{rms}$ \\
\hline Dark current, $\mathrm{D}$ & $40 \mathrm{e}-/ \mathrm{s}$ \\
\hline Exposition time, $\mathrm{t}$ & $70 \mu \mathrm{s}-67 \mathrm{~s}$ \\
\hline Effective area, $\mathrm{A}_{\mathrm{eff}}$ & $15 \mathrm{~mm} \times 15 \mathrm{~mm}$ \\
\hline Pixel area, $\mathrm{A}_{\text {pix }}$ & $7.4 \mu \mathrm{m} \times 7.4 \mu \mathrm{m}$ \\
\hline
\end{tabular}

* The read noise includes the shot noise as well as the electronic noise

Using these parameters, the dynamic range (DR), the signal-to-noise ratio (SNR) at full well capacity, $\mathrm{SNR}_{\max }$, and the minimum and maximum measurable power incident on the CCD, $\mathrm{P}_{\min }$ and $\mathrm{P}_{\max }$, can be calculated. The equations for the dynamic range and the SNR are as follow:

$$
\begin{gathered}
D R=\frac{F W}{n_{R}} \\
S N R=\frac{\Phi \cdot \eta \cdot t}{\sqrt{\Phi \cdot \eta \cdot t+D \cdot t+n_{R}}}
\end{gathered}
$$

$\Phi$ is the number of incident photons per second and $t$ is the exposition time in second. For the results shown in Table 5.6, the minimum exposition time, $70 \mu \mathrm{s}$, is used. $\Phi \cdot \eta \cdot t$ is equivalent to the number of electrons $\left(n_{e}\right)$ generated for a given input signal. Equation 5.8 is used to do the conversion from number of generated electrons to incident power $\mathrm{P}$.

$$
P=h v \Phi=h v \frac{n_{e}}{\eta t}
$$


Where $h$ is Planck's constant and $v$ is the frequency obtained from $\mathrm{c} / \lambda$, c being the speed of light in vacuum and $\lambda$ the wavelength. The maximum power allowed on one pixel $\left(\mathrm{P}_{\max }\right)$ is calculated using equation 5.8 with $\mathrm{n}_{\mathrm{e}}$ being the full-well capacity. The minimum power allowed on one pixel $\left(\mathrm{P}_{\min }\right)$ is calculated with the same equation but $\mathrm{n}_{\mathrm{e}}$ in this case is the number of electrons corresponding to a SNR of 2 as this corresponds to the lowest detectable signal. For this sensor, a SNR of 2 is obtained with 20 electrons. Table 5.6 gives the performance of the sensor.

Table 5.6 - Performance of the Kodak sensor

\begin{tabular}{|l|l|}
\hline Performance & F-421B / KAI-04022 \\
\hline $\mathrm{DR}$ & $4444(72 \mathrm{~dB})$ \\
\hline $\mathrm{SNR}_{\max }$ & $200(46 \mathrm{~dB})$ \\
\hline $\mathrm{P}_{\max }$ & $0.53 \mathrm{nW}$ \\
\hline $\mathrm{P}_{\min }$ & $0.265 \mathrm{pW}$ \\
\hline
\end{tabular}

The incident power depends on the exposition time and therefore as the exposition time increase, the maximum and minimum power decrease. To verify that the chosen light source has enough power to produce a measurable response on the $\mathrm{CCD}$, the incident power on one pixel can be estimated. The source has an output power of $5.2 \mathrm{~mW}$ and the following losses are assumed: $3 \mathrm{~dB}$ loss from the splitting ratio, $3 \mathrm{~dB}$ loss from the fibre coupler, $3 \mathrm{~dB}$ loss through the polariser and $3 \mathrm{~dB}$ loss through the optical system. The incident power, $\mathrm{P}_{\mathrm{s}}$, obtained is $0.325 \mathrm{~mW}$. To calculate the incident power from the source on one pixel, the result is multiplied by the pixel area and divided by the sensor effective area (assuming a full coverage of the CCD).

$$
P_{\text {inc }}=\frac{P_{s}}{A_{\text {eff }}} A_{p i x}
$$


Using $A_{\text {pix }}=54.76 \mu \mathrm{m}^{2}$ and $A_{\text {eff }}=225 \mathrm{~mm}^{2}, P_{\text {inc }}$ is found to be $79.1 \mathrm{pW}$. This is in between the maximum and minimum detectable power of the CCD and therefore the light source is appropriate for the cameras.

\subsubsection{Optical Components}

As mentioned earlier, the large core of the fibre brings a challenge for the optical system as the light coming out of the fibre cannot be collimated perfectly. In fact, the source has to be treated as an extended source, meaning that each point across the fibre core acts as a different point light source. Figure 5.6 demonstrates how the rays travel though a collimating lens in the case of an extended light source. The rays coming from the center of the core (red) will be collimated properly but the rays coming from the top of the fibre (green), for example, will exit the collimating lens with an angle $\theta$. This angle is calculated with equation 5.10 where $\mathrm{y}_{0}$ is the radius of the fibre core and $\mathrm{f}$ is the focal length of the collimating lens. A longer focal length will give a smaller angle and thus a better collimation.

$$
\tan \theta=\frac{-1}{f} y_{0}
$$

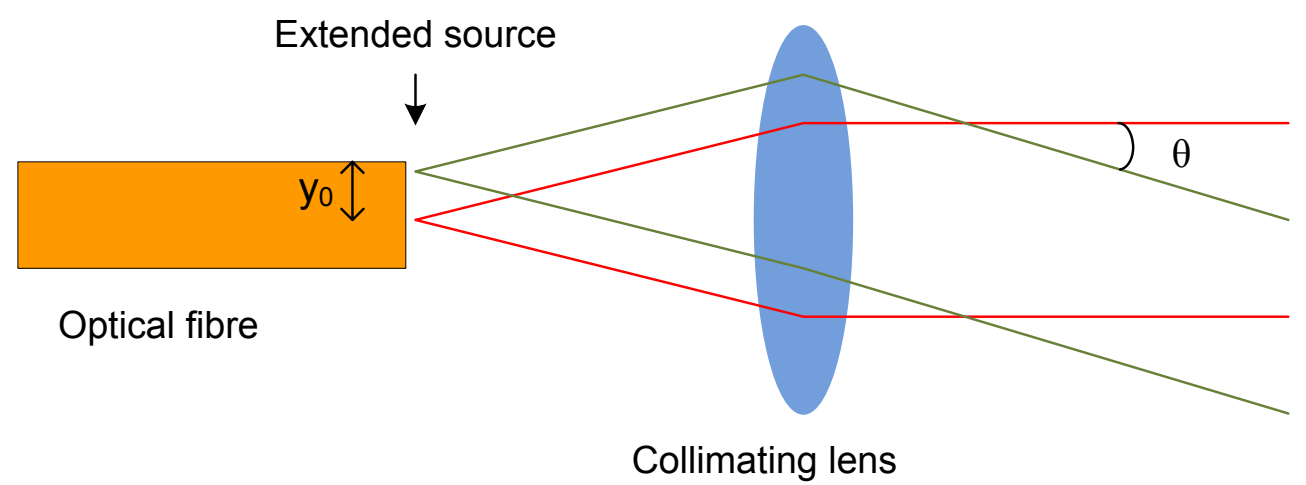

Figure 5.6 - Ray tracing for an extended source (multimode optical fibre) 
This collimating issue will blur the SPR dip on the cameras since the rays will not be incident on the gold film with all the same angles and the incident angle on the grating will vary as well which will blur the wavelength diffraction. The blurring in the angular dimension is shown in Figure 5.7 where two rays (in red) reflecting off the gold film at the same angle will not end up on the same pixel on the CCD camera. This problem can be taken care of by using two lenses, a focusing lens and a collimating lens. This will achieve two purposes at the same time. The lenses will be used to resize the beam such that it fit properly on the sensor of the camera. Also, it will help the blurring as the rays with identical angular content will overlap at the focal point of the second lens. By placing the camera at the focus, the blurring will be minimized.

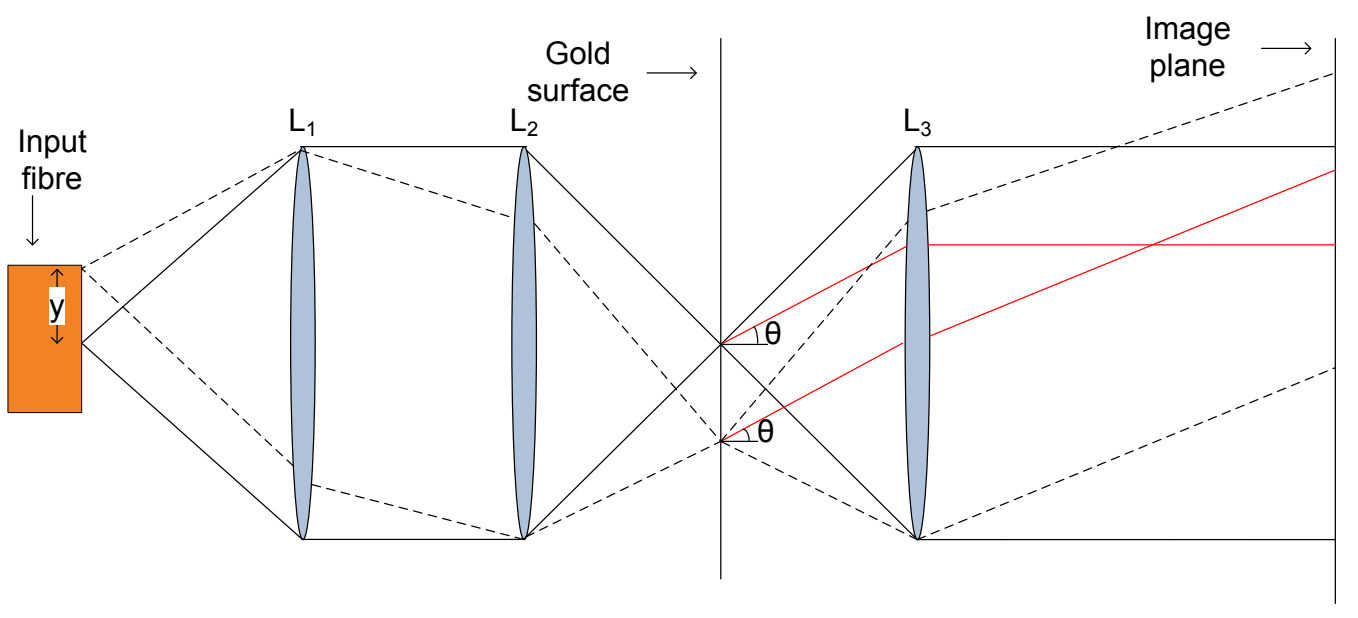

Figure 5.7 - Effect of the collimation of an extended source on the angular resolution at the image plane. $L_{1}$ is a spherical collimating lens and $L_{2}$ and $L_{3}$ are identical spherical focusing and collimating lenses.

The wavelength blurring is a bigger challenge since the only thing that can be done to minimize it is to increase the initial beam size (through a long focal length collimating lens). The wavelengths are blurred because the rays are focused on the cameras according to their reflection angles on the grating which depends on the incident angle. If the rays from the same wavelength do not hit the grating with 
the same angle, they will reflect at different angles and will end up on different pixels on the camera. Therefore the initial beam has to be as large as possible.

Simulations were performed to see how the blurring will affect the refractive index estimation. Code $\mathrm{V}$ was used to simulate the beam traveling through the optical components and estimate the blurring while Matlab was used to simulate the appropriate blurring on the simulated SPR images. Considering an input beam with a $20 \mathrm{~mm}$ diameter (achieved using a collimating lens with a focal length of $25 \mathrm{~mm}$ ), the overlap between rays would be $3 \%$ in the angular dimension and $46 \%$ in the spectral dimension. The angular blurring can therefore be solved such that the data is good enough to be used. On the other hand, the blurring with the wavelengths is quite high even with a large beam. However, when looking at the image it produces, it can be seen that the effect will not be as great as if the blurring in the angular dimension was large. This is the case because the main feature for the estimation is the sharpness of the dip and its displacement with respect to angles. Figure 5.8 demonstrates an ideal image and Figure 5.9 illustrates an image blurred with a Gaussian function with $3 \%$ blurring in the angular dimension and $46 \%$ blurring in the wavelength dimension. The difference between the two images is that the dip line is flared at the top and bottom on the blurred image. Using the data processing technique on the blurred images, the simulation showed that the refractive index estimation was still possible and that the results were good as it is detailed in Table 5.7. In the absence of noise, the estimation of the refractive index change is the same as before with a negligible error. When noise is added (4-bit of noise in this case), the error is a little bit bigger than without any blur but the results are comparable and stay within the same order of magnitude. Therefore, the system design is suitable to implement the biosensor. 


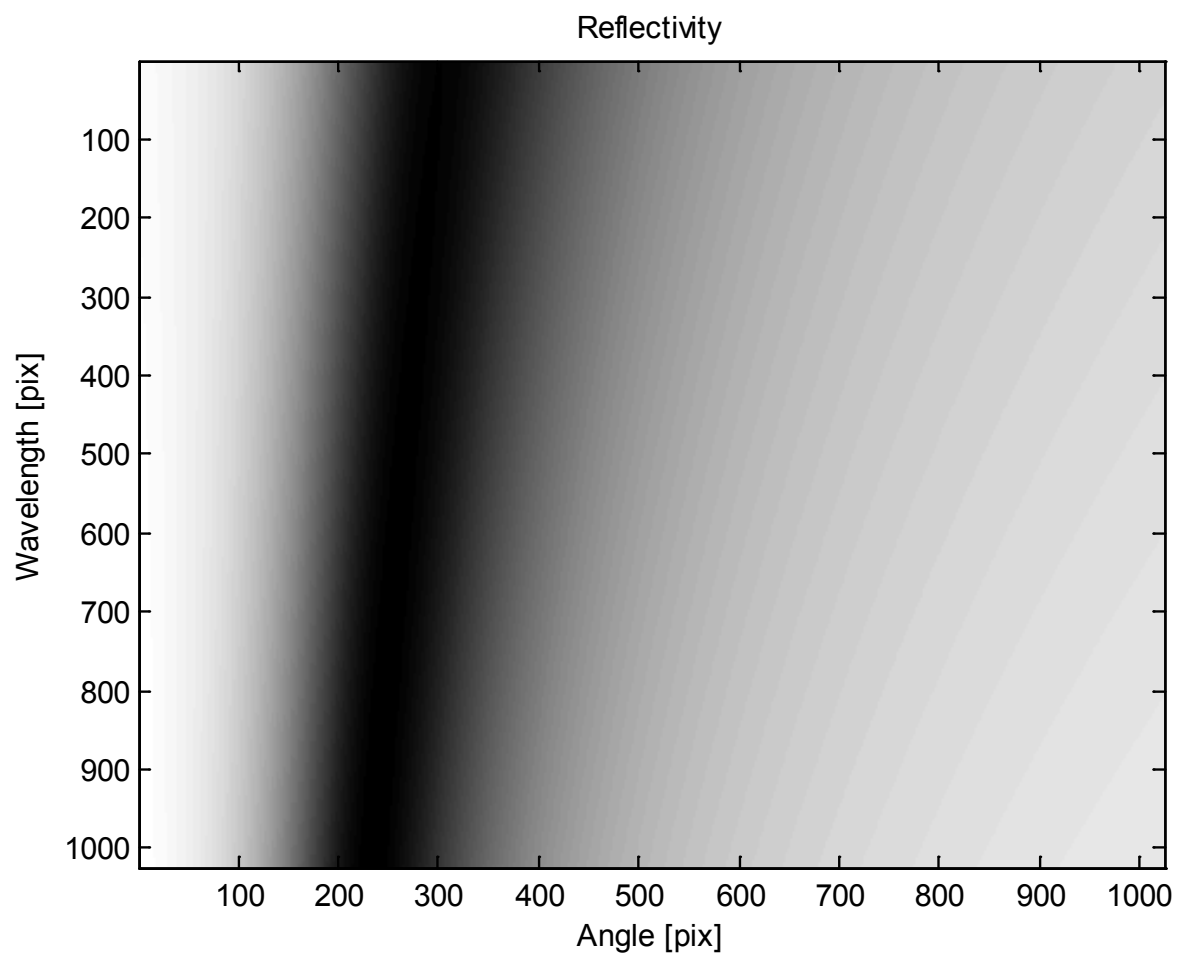

Figure 5.8 - A 1024 x 1024 ideal SPR image

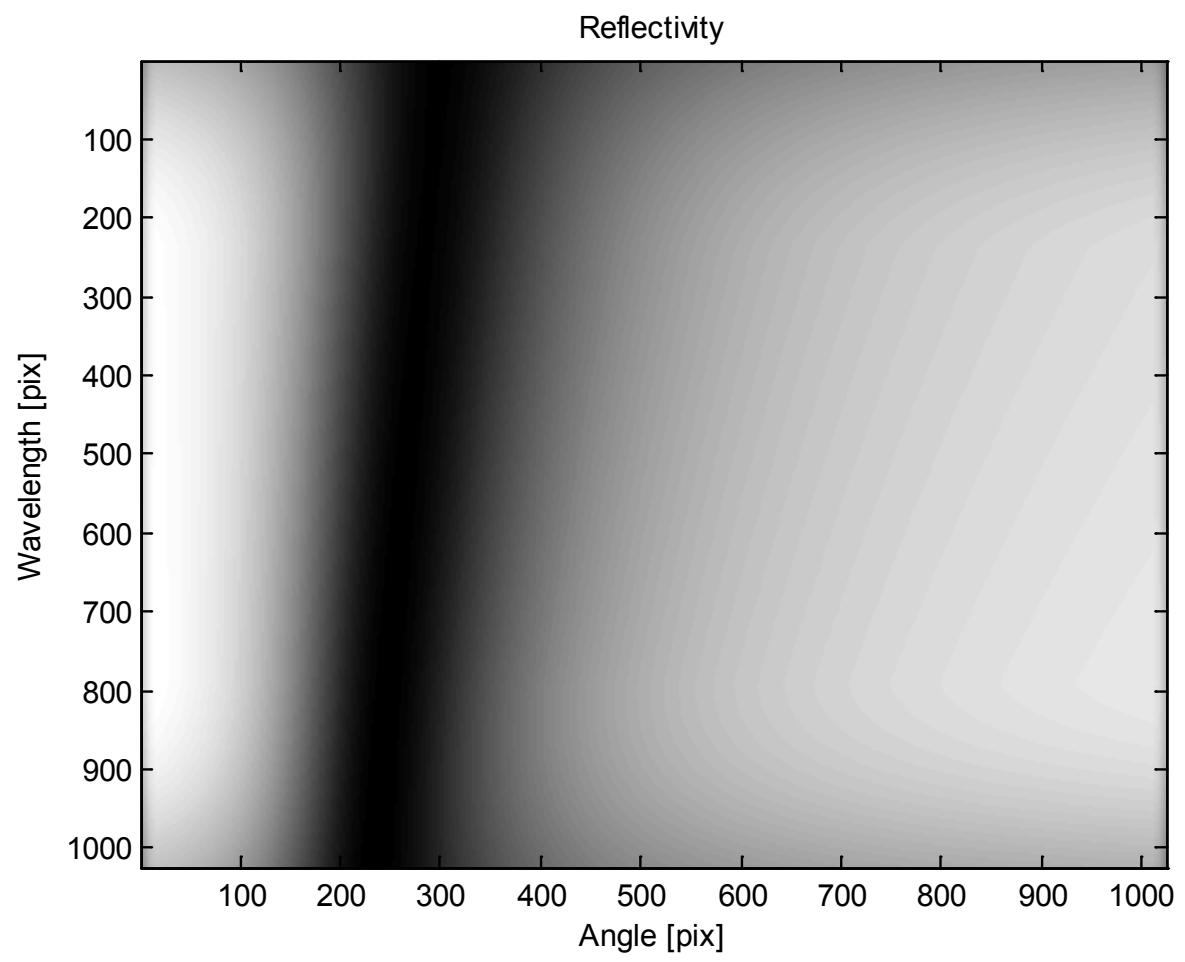

Figure 5.9 - A 1024 x 1024 blurred SPR image 
Table 5.7 - Theoretical RMS estimation error for blurred 1024x1024 SPR images at a center wavelength of $630 \mathrm{~nm}$. 14-bit of quantization noise is considered in both the noise-free and noisy cases.

\begin{tabular}{|l|c|c|}
\hline $\begin{array}{l}\text { RI } \\
\text { change } \\
(\boldsymbol{\Delta})\end{array}$ & $\begin{array}{l}\text { RMS Estimation Error } \\
\text { (no noise) }\end{array}$ & $\begin{array}{l}\text { RMS Estimation Error } \\
\text { (4-bit noise) }\end{array}$ \\
\hline $10^{-6}$ & $5.77 \mathrm{e}-9$ & $4.45 \mathrm{e}-8$ \\
\hline $10^{-7}$ & $1.06 \mathrm{e}-16$ & $5.65 \mathrm{e}-8$ \\
\hline $10^{-8}$ & $1.36 \mathrm{e}-16$ & $4.33 \mathrm{e}-8$ \\
\hline
\end{tabular}

An appropriate beam size was chosen such that the collimation would be optimised and the beam would still fit in an optical cage system. A beam size around $20 \mathrm{~mm}$ was found convenient and it can be obtained by using a lens with a focal length of $25.4 \mathrm{~mm}$. Once the beam is collimated, the light has to be focused on the sensing surface at the right angles. The range of angles was chosen such that it includes the resonance for the buffer that will be commonly used with the biosensor as well as to cover an appropriate dynamic range. For a wavelength range around $630 \mathrm{~nm}$ and water as the buffer, the range of angles chosen was $53^{\circ}$ to $61^{\circ}$. With this range, the SPR dip for water is at the left of the image and a range of $8^{\circ}$ allows a change in refractive index of about 0.07 RIU to be measured. To achieve this range of angles, a focusing lens with a focal length of $75 \mathrm{~mm}$ is required. In the previous design, a cylindrical lens was used; but in this case, a spherical lens is needed since the beam is large and the light spot on the gold should be as small as possible to fit in the chambers. The $20 \mathrm{~mm}$ beam has to be focused in all directions to become a point on the sensing surface that fits in a 5 $\mathrm{mm}$ diameter chambers. It does not affect the results if the beam is not perpendicular in the wavelength dimension because only TM polarised light will excite surface plasmons. 
The prism is used to enhance the momentum of the incoming photons and it will also determine the angular range with the focusing lens used. The range of angles incident on the gold will be smaller with the use of the prism than if the reflection was happening in air. Figure 5.10 shows the bending of the rays going through the prism. The range of angles can be calculated using Snell's law:

$$
n_{i} \sin \theta_{i}=n_{o} \sin \theta_{o}
$$

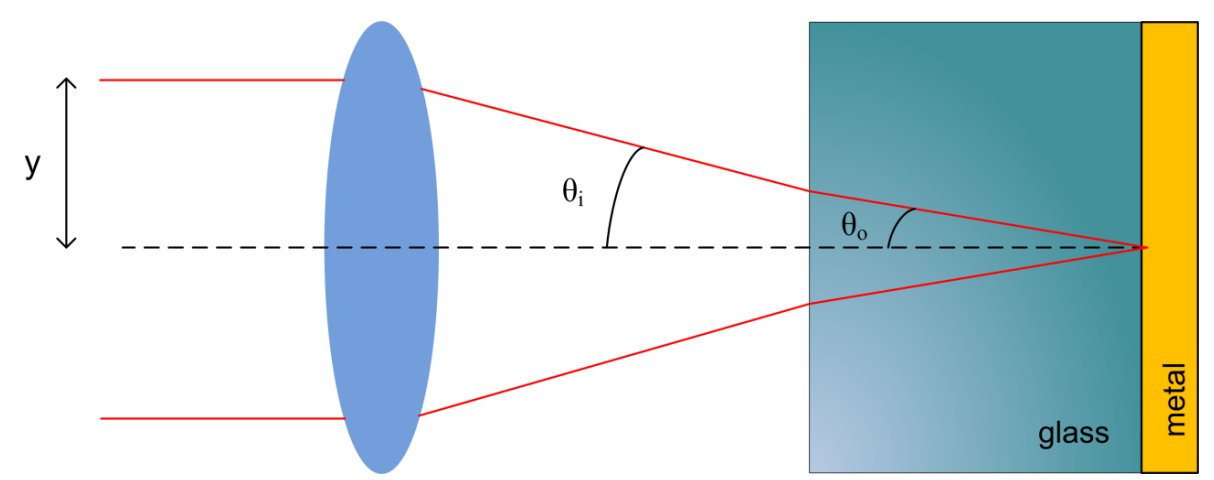

Figure 5.10 - Rays bending due to the change in the surrounding refractive index

The output angle, $\theta_{\mathrm{o}}$, is determined by the input angle with respect to the normal of the interface, $\theta_{i}$, the refractive index of the initial side of the interface, $n_{i}$, and the refractive index of the output side of the interface, $\mathrm{n}_{0}$. The angular range produced by the focusing lens is $2 \theta=\tan ^{-1}(y / f)$ where $y$ is the half size of the beam and $\mathrm{f}$ is the focal length of the focusing lens. In this case, $\mathrm{y}$ is $20 \mathrm{~mm}$ and $\mathrm{f}$ is $75 \mathrm{~mm}$ which give an angle of $7.5^{\circ}$ and therefore an angular range of $15^{\circ}$. When going through the prism, the rays will bend and the angular range will decrease. The refractive index of SF11 glass is 1.779 RIU at a wavelength of 630 $\mathrm{nm}$. The output angle, given an incident angle of $7.5^{\circ}$, will be $4.2^{\circ}$ which then gives a full angular range of $8.4^{\circ}$.

The prism used in this system is a $25 \mathrm{~mm}$ SF11 glass equilateral triangular prism. The beam goes through one side of the triangle and reflects on the gold with the 
specific angles allowing resonance for water, $53^{\circ}$ to $61^{\circ}$. The glass must have a triangular shape because the large angles required for the surface plasmon resonance to occur would totally reflect on a flat piece of glass. With an equilateral triangular shape, the rays are incident on the side with a small angle with respect to the normal of the glass while reflecting on the gold with the appropriate angles. Figure 5.11 demonstrates how the rays are incident on the glass.

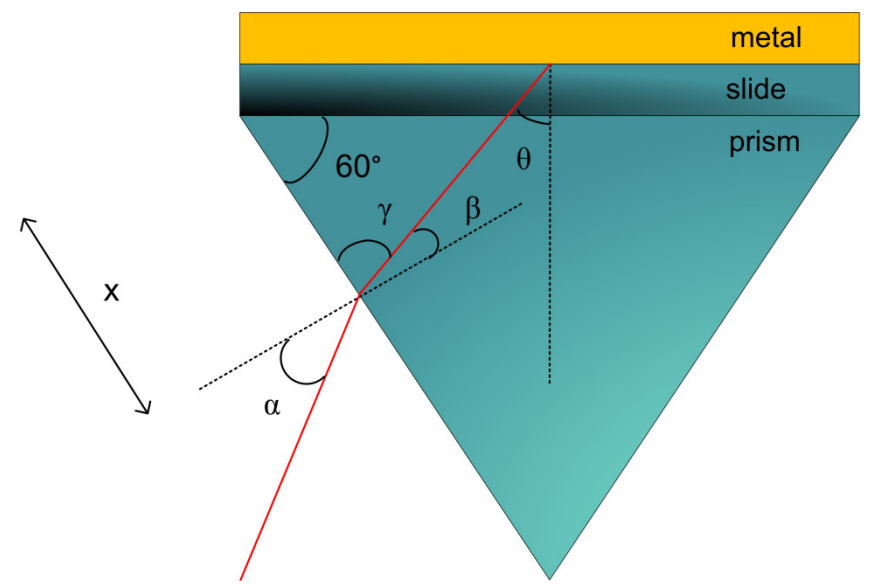

Figure 5.11 - Rays entering the triangular prism with an angle $\alpha$ and reflecting on the gold with an angle $\theta$

Using the ray in the middle of the beam, the angle $\theta$ has to be $57^{\circ}$. Since the triangle is equilateral, the corners of the prism are all $60^{\circ}$ and thus angle $\gamma$ is $87^{\circ}$. Following, the angle $\beta$ is $3^{\circ}$ and using Snell's law, angle $\alpha$ is found to be $5.3^{\circ}$. The next important parameter is the point on the glass where the rays are incident such that they reflect in the middle of the prism. The rays have to reflect in the middle because this is where the sensing region is. The gold is not deposited directly on the prism but on a glass slide that is placed adjacent to the prism using a refractive index matching liquid to eliminate any air pockets on the interface that would reflect the rays. The thickness of the glass slide is $1.6 \mathrm{~mm}$ and has to be taken into account for the position of the incident point on the side of the prism. Using Figure 5.11 we can calculate $\mathrm{x}$, the distance from the corner of the prism to the point of incidence and find $5.7 \mathrm{~mm}$. 
After the reflection on the gold, the beam is re-collimated with an identical spherical lens with a focal length of $75 \mathrm{~mm}$. Then a grating and a focusing cylindrical lens are used to diffract the wavelengths and focus the rays onto the camera. Figure 5.12 illustrates this concept. The combination grating-lens is chosen to cover an area as large as possible on the camera while achieving incident and reflected angles on the grating that are physically implementable. The reflected angles are calculated using equation 5.12 where $a$ is the grating period (or the spacing between the grooves), $\theta_{\mathrm{i}}$ is the incident angle, $\theta_{\mathrm{r}}$ is the reflected angle and $m$ is the reflection order.

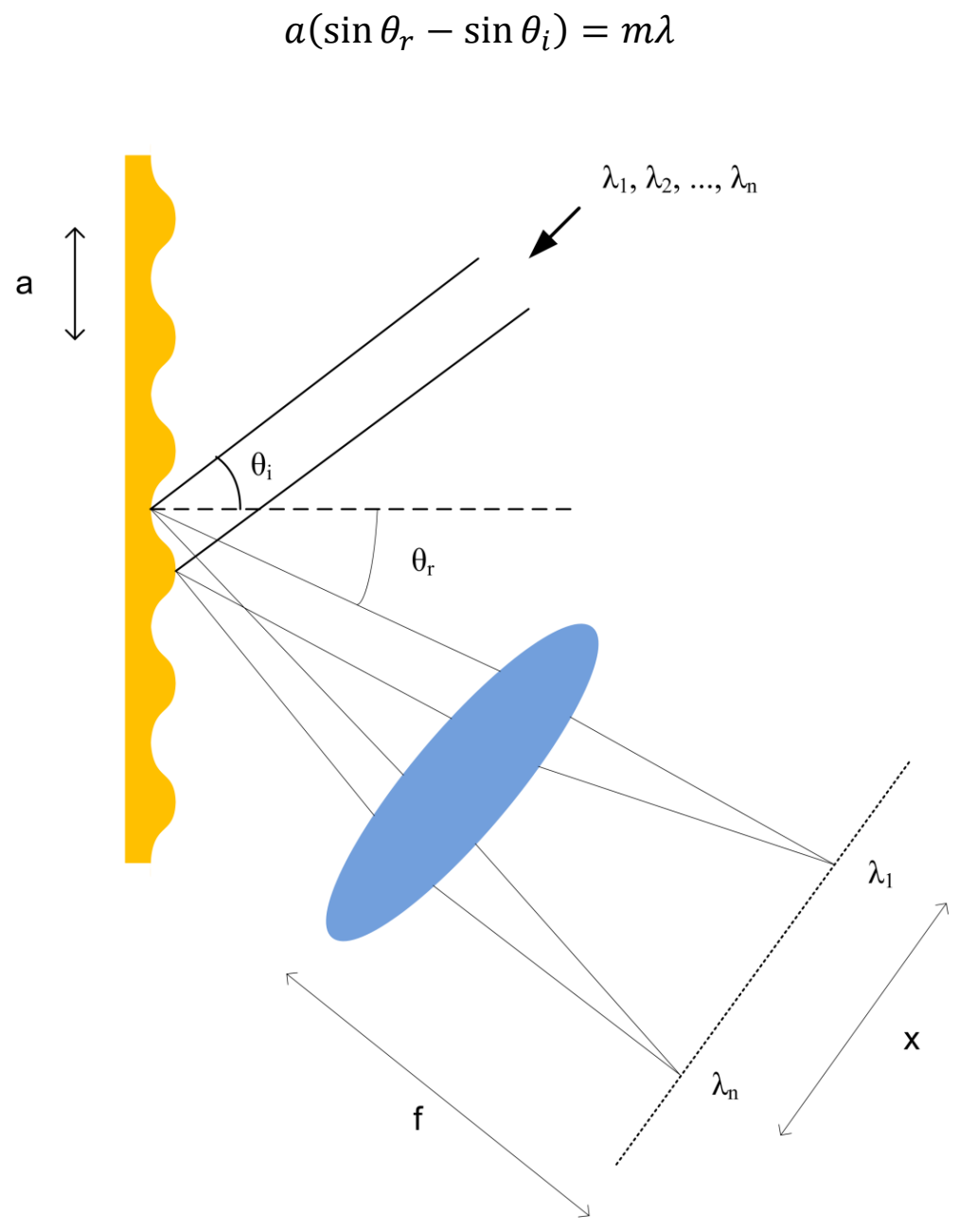

Figure 5.12 - Diffraction of an incident beam on a grating and focusing onto an image plane with a lens 
The size of the image on the CCD, $\mathrm{x}$, depends on the bandwidth of the source, the focal length, $f$, of the focusing lens, the incident angle as well as the grating period. Equation 5.13 gives the position of the rays for one wavelength with respect to the central axis of the lens and equation 5.14 gives the image size on the $\mathrm{CCD}$ which is the difference between the position for the longer wavelength and the position for the shorter wavelength.

$$
\begin{gathered}
x_{k}=f \cdot \tan \left(\theta_{r k}\right)=f \cdot \tan \left(\sin \left(\frac{m \lambda_{k}}{a}+\sin \theta_{i}\right)\right) \\
x=\left|x_{n}-x_{1}\right|=\left|f \cdot\left(\tan \left(\theta_{r n}\right)-\tan \left(\theta_{r 1}\right)\right)\right|
\end{gathered}
$$

The components chosen for this work are a grating with a period of $0.556 \mu \mathrm{m}$ (1800 grooves per $\mathrm{mm}$ ) and a cylindrical lens with a focal length of $150 \mathrm{~mm}$. Using the order $\mathrm{m}=-1$ and an incident angle of $20^{\circ}$, the reflected angles are from $50.884^{\circ}$ (shorter wavelength) to $-53.910^{\circ}$ (longer wavelength). The minus sign means that the rays reflect on the same side of the grating normal as the incident rays. There is a trade-off in choosing an appropriate grating. The spacing between the reflected angles of each wavelength increase as the number of grooves per $\mathrm{mm}$ gets larger. On the other hand, given a wavelength in the visible spectrum and a large number of grooves per $\mathrm{mm}$, the order $\mathrm{m}=-1$ must be used as the order $\mathrm{m}=1$ does not exist in that case. By using the order $m=-1$ the reflected angles are on the same side as the incident angles and the angle must be chosen properly such that there is an appropriate difference between the incident and reflected rays. A smaller number of grooves per mm would have been more suitable for the implementation as the order $m=1$ could have been used but the coverage of the CCD would have been much smaller. Since the order $m=-1$ is used in this case, the incident angle was chosen to give a difference of $30^{\circ}$ between the incident and reflected beams. Given the diffraction angles and the focal length, the size of the image on the CCD for the wavelength dimension is $7.92 \mathrm{~mm}$. 
The last optical components before the cameras are two lenses used both to modify the size of the beam in the angle dimension to cover a good portion of the $\mathrm{CCD}$ and to solve as much as possible the problem with the angular blurring. The lenses chosen are cylindrical lenses with focal lengths of $38.1 \mathrm{~mm}$ and $25.4 \mathrm{~mm}$. It will therefore reduce the beam size by a third, from $20 \mathrm{~mm}$ to $13 \mathrm{~mm}$ which is appropriate for a $15 \mathrm{~mm} \mathrm{CCD} \mathrm{camera.}$

\subsubsection{Prism Holder and Fluidic System}

In order to physically hold the prism and the glass slide coated with gold as well as introduce fluid in the sensing chambers, a prism holder and a fluidic system have to be fabricated. The requirements are that the prism must be held in place with the least obstruction and that two sealed chambers must be taking place on the gold (one for the signal and one for the reference). Also, there must be space for a TEC on top of the chambers to compensate for the increase in temperature as well as a heat sink which is essential with the use of a Peltier module. In and out tubes to and from the chambers are necessary to distribute the fluid in the chambers. A pump is used to ensure a constant flow of the buffer and introduce the specific samples. Figure 5.13 shows a side view through the prism holder and fluidic system block. The second chamber and set of tubes are behind the one shown on the figure. A single Peltier module covers both chambers. 


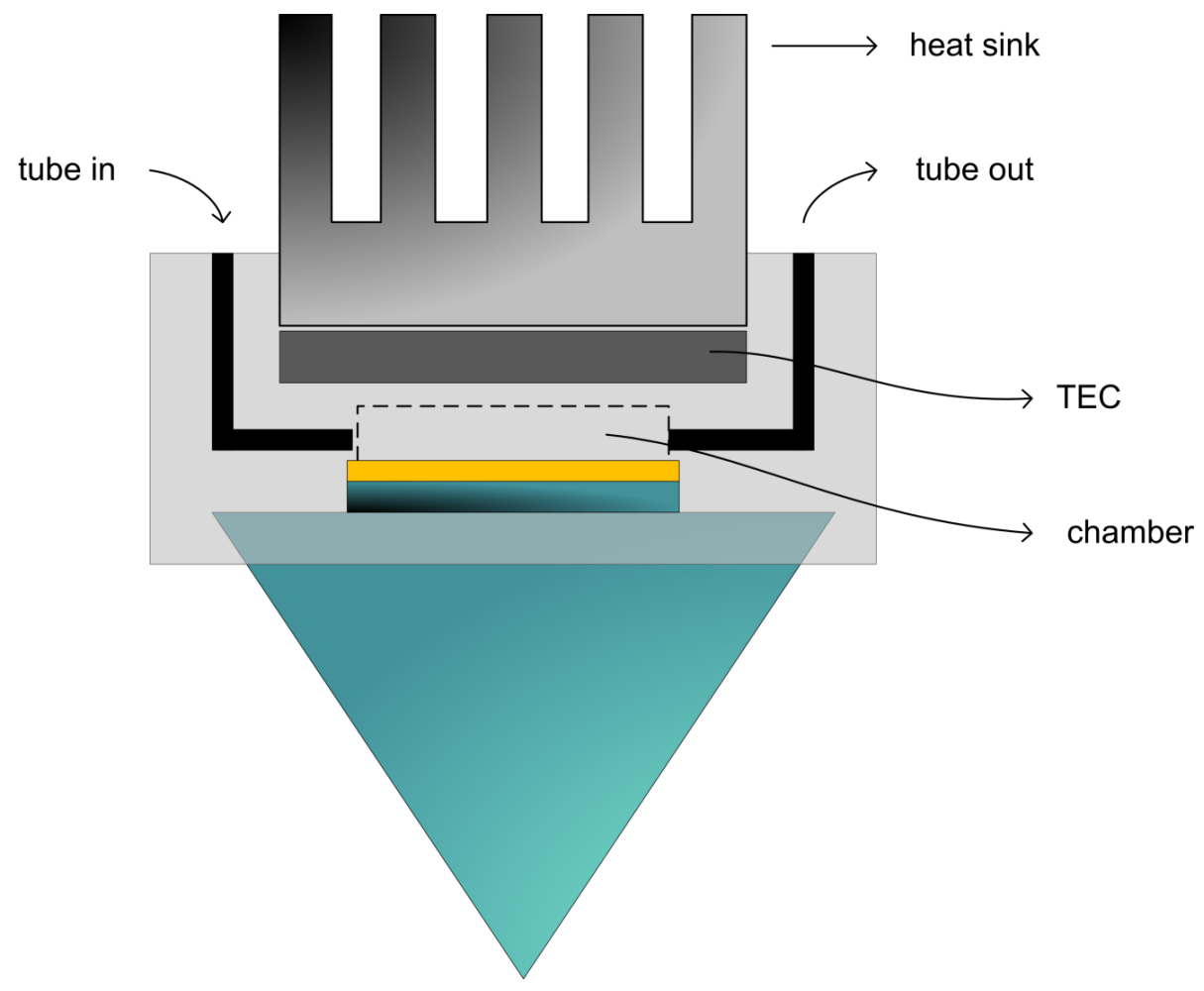

Figure 5.13 - Prism holder and fluidic system

\subsection{Interface and Image Analysis}

Beyond the optical system, the data analysis is a central element in estimating the refractive index of the sample under study. In between the optical system and the software, the cameras are the devices converting the light intensity into digital data for the analysis to take place. The refractive index estimation depends highly on the quality of the images taken and the rate at which those images are acquired. As mentioned before, high quality data is the key for a low detection limit.

The main feature required by the acquisition software, which was programmed with National Instruments LabVIEW, is the synchronisation of the cameras. In order to take full advantage of using a reference channel, the two cameras must acquire images at the same time. This ensures that the fluctuations in the source and the temperature changes are equal in both images so that they can be compensated as much as possible. The best way to synchronise the cameras is to 
set them in master-slave mode. One camera, the master camera, receives a signal from the acquisition software to trigger the acquisition while the second camera, the slave, is triggered by the master camera through hardware connections. The delay between the data acquisition on the cameras is then as small as it can be and is around $11 \mu \mathrm{s}$. This is an improvement over the previous design where the reference images were $33 \mathrm{~ms}$ delayed with respect to the signal images. The onboard memory prevents any loss of data since the transfer of the images to the computer cannot be done from both cameras at the same time.

The acquisition is continuous and the images are processed as they are saved on the computer to obtain real-time measurements. To stay in real-time throughout the entire time the measurements take place, the acquisition time on the computer must match the processing time. Otherwise, the processing falls behind and the delay between the acquisition of an image and the results increases continuously. The solution is to average images over a period of time corresponding to the processing time. By doing so, no images are discarded and all the information is kept for the analysis. Also, averaging reduces the noise in the data and therefore increases the quality of the images. The on-board averaging is the best way to average images; only one image is transferred to the computer and saved. Otherwise, the averaging must be done by software which increases the processing time because the write and read operations are time consuming and too many of those operations would have to be performed. The cameras purchased from Allied Vision Technologies are equipped with on-board averaging. The number of images to average is left to be determined and will depend on the processing time. 


\section{Chapter 6: Conclusion}

\subsection{Summary}

In this thesis, it was demonstrated that the spectro-angular surface plasmon resonance biosensor previously designed could be modified and would benefit to work in the visible spectrum instead of the near infra-red spectrum. The high resolution and the high precision cameras allow high quality images to be acquired; those high quality images are required by the data processing technique in order to reduce the detection limit of the biosensor since the resolution and the precision of the data greatly influence the accuracy of the refractive index estimation with the Double Projection Method. New cameras were purchased for the experimental set-up with a resolution of $2048 \times 2048$ pixels and a data precision of 14 bits. This is a significant improvement with respect to the previous cameras which had a resolution of 480x640 pixels and a data precision of 10 bits. In terms of the detection limit, the theoretical resolution of the biosensor was evaluated to improve from $3.4 \times 10^{-8} \mathrm{RIU}$ to practically zero for noise-free data and from $5.8 \times 10^{-7}$ RIU to $3.9 \times 10^{-8}$ RIU in the presence of noise for a refractive index change of $10^{-6}$ RIU.

A new experimental system has been completely designed and was presented in this thesis. The simulations based on the parameters of the experimental set-up have shown that it was possible to implement the system in the visible spectrum and to deal with the challenges associated with the parameters of the light source chosen. All the required components for the new biosensor have been bought and the fabrication has started. It is expected that the detection limit of the new experimental system will be lower than the one of the previously built biosensor which was $1.5 \times 10^{-6} \mathrm{RIU}$. 


\subsection{Future Work}

\subsubsection{Fabrication and Testing of the Experimental Set-Up}

The new system is being built and important tests will be performed when the fabrication is finished. The first test to be done is a simple refractive index change. A refractive index change in the order of $10^{-2}$ or $10^{-3}$ RIU will help test if the system is working. It must be verified that the parameters of the output images, such as the ranges of angles and wavelengths, are the ones that were expected. It can be done by analysing the images for samples of known refractive indices across the dynamic range of the biosensor. Adjustment can be made to either the optical system or the parameters of the basis for the data processing. The main assessment with a new system is to calibrate it with multiple known refractive index changes of different order of magnitude to validate that the optical system and the data processing software are estimating the changes accurately.

Once the system is found to work properly, the detection limit of the biosensor will be determined with the measured noise level in the data. The performance will be compared with the previous system to demonstrate any improvement and validate the theory. Finally, relevant assays with appropriate proteins and other biomolecules will be performed in order to recognize the system as a valid biosensor.

\subsubsection{One Camera For Both SPR Channels}

Another step once the biosensor is working would be to modify the output of the system such that only one camera is used to read both SPR channels. The resolution of the cameras chosen is large enough to be divided in two so that two optical beams can be read on the same sensor. This would make the data acquisition much simpler. There is no need for a multi-camera synchronisation 
and therefore no delay between the signal and reference images. Only one image is acquired and then divided in two to separate the data from both channels.

It is possible to implement with the current design of the system since two identical but separate optical path are set-up for each channel. In the previous design, both channels were sharing most of the same lenses and the grating. With two beams reflecting on a single grating with the same angle, it is not possible to focus them on the same cameras. The distance between the images cannot be smaller than the width of the output cylindrical lenses used to focus the beams in the wavelength dimension and this width is greater than the size of the sensor of the camera. However when two gratings are used, the beams can be redirected towards the same camera symmetrically such that there is a minimum optical path difference between both channels.

This idea brings another interesting option which could be implemented: multiplexing. A single camera could be used for multiple channels. The size of the system is reduced by the use of only one camera instead of one for each channels and the synchronisation between multiple cameras is not necessary. On the other hand, it would be a challenge to image multiple beams on the same sensor. Also the number of pixels required for one channel for an accurate refractive index estimation would have to be determined in order to know how many channels can be implemented for the data to be acquired with the same device.

\subsubsection{Normalisation with Polarisation}

Finally, another modification that could be done on the system is to use the TE polarisation to normalise the data. The polariser at the entrance of the optical path could be removed such that both TE and TM polarisations are present in the beams. Only TM polarised light excites surface plasmons. Before the camera, the beam could be split in TE and TM polarisations and both beams would be imaged on the same camera. The data from the TE polarised light could be used to 
normalise the data from the TM polarised light that shows an SPR dip. The normalisation in the current system is done with an image saved at the beginning of an assay by acquiring the data when the buffer is not yet in the chamber. There is no SPR response with air since the range of angles does not cover the resonance conditions of this sample. The normalisation with TE in real-time would help reduce the noise from power fluctuations in the source and the background noise as well as any flaws in the images due to the optical system. In the current work, the normalisation with air can compensate for the last point but the fluctuations are taken care of by the reference channel.

In addition to this normalisation with TE, both the signal and reference channels, could be image on the same camera alternatively. Both channels could be imaged on the full CCD and a shutter could be used to alternate between the channels. In this case, the normalisation would compensate the intensity fluctuations and background noise while the reference channel would compensate the temperature fluctuations. The fact that both images are not acquired at the same time but alternatively means that high frequency noise could not be fully compensated but since the normalisation would be synchronised, it is already taken care of. The temperature fluctuation is a slower process and therefore the lack of synchronisation would not be an issue. 


\section{References}

[1] V. Seena et al., "Fabrication and characterization of novel polymer composite microcantilever sensors for explosive detection," in Proceedings of the IEEE International Conference on Micro Electro Mechanical Systems (MEMS), vol. 6, Wanchai, Hong Kong, 2010, pp. 523-529.

[2] V. Seena, A. Rajorya, P. Pant, S. Mukherji, and V.R. Rao, "Polymer microcantilever biochemical sensors with integrated polymer composites for electrical detection," Solid State Sci., vol. 11, no. 9, pp. 1606-1611, 2009.

[3] A. Tuantranont, A. Wisitsora-at, P. Sritongkham, and K. Jaruwongrungsee, "A review of monolithic multichannel quartz crystal microbalance: a review," Anal. Chim. Acta, vol. 687, no. 2, pp. 114-128, 2011.

[4] S.-E. Oh, S.H.A. Hassan, and S.W. Van Ginkel, "A novel biosensor for detecting toxicity in water using sulfur-oxidizing bacteria," Sens. Actuators, $B$, vol. 154, no. 1, pp. 17-21, 2011.

[5] C. Staii, A. T. Johnson Jr, M. Chen, and A. Gelperin, "DNA-Decorated Carbon Nanotubes for Chemical Sensing," Mater. Res. Soc. Symp. Proc., vol. 963, pp. 301-307, 2006.

[6] R. Irawan et al., "Polymer waveguide sensor for early diagnostic and wellness monitoring," Biosens. Bioelectron., vol. 26, no. 8, pp. 3666-3669, 2011.

[7] R. Bruck, E. Melnik, P. Muellner, R. Hainberger, and M. Lämmerhofer, "Integrated polymer-based Mach-Zehnder interferometer label-free streptavidin biosensor compatible with injection molding," Biosens. Bioelectron, vol. 26, no. 9, pp. 3832-3837, 2011.

[8] C. Wu, Y. Zhou, X. Miao, and L. Ling, "A novel fluorescent biosensor for sequence-specific recognition of doublestranded DNA with the platform of graphene oxide," Analyst, vol. 136, pp. 2106-2110, 2011.

[9] P. Schubert-Ullrich et al., "Commercialized rapid immunoanalytical tests for determination of allergenic food proteins: an overview," Anal. Bioanal. Chem., vol. 395, no. 1, pp. 69-81, 2009.

[10] L. Monaci, M. Brohée, V. Tregoat, and A. Van Hengel, "Influence of baking time and matrix effects on the detection of milk allergens in cookie model food system by ELISA," Food Chem., vol. 127, no. 2, pp. 669-675, 2011.

[11] J. Homola, S. S. Yee, and G. Gauglitz, "Surface plasmon resonance sensors: review," Sens. Actuators, B, vol. 54, no. 1, pp. 3-15, 1999.

[12] C. Nylander, B. Liedberg, and T. Lind, "Gas detection by means of surface plasmon resonance," Sens. Actuators, vol. 3, no. C, pp. 79-88, 1982.

[13] B. Liedberg, C. Nylander, and I. Lunström, "Surface plasmon resonance for gas detection and biosensing," Sens. Actuators, vol. 4, no. C, pp. 299-304, 1983.

[14] C. J. Alleyne, A. G. Kirk, W.-Y. Chien, and P. G. Charette, "Numerical 
method for high accuracy index of refraction estimation for spectro-angular surface plasmon resonance systems," Opt. Express, vol. 16, no. 24, pp. 19493-19503, 2008.

[15] C. J. Alleyne, P. J. R. Roche, S. Filion-Côté, and A. G. Kirk, "Analysis of surface plasmon spectro-angular reflectance spectrum: real-time measurement, resolution limits, and applications to biosensing," Opt. Lett., vol. 36, no. 1, pp. 46-48, 2011.

[16] P.B. Johnson and R. W. Christy, "Optical constants of the noble metals," Phys. Rev. B, vol. 6, no. 12, pp. 4370-4379, 1972.

[17] B. Sepúlveda, A. Calle, L.M. Lechuga, and G. Armelles, "Highly sensitive detection of biomolecules with the magneto-optic surface-plasmon-resonance sensor," Opt. Lett., vol. 31, no. 8, pp. 1085-1087, 2006.

[18] J. Homola, "Present and future of surface plasmon resonance biosensors," Anal. Bioanal. Chem., vol. 377, no. 3, pp. 528-539, 2003.

[19] SCHOTT. (2011) SCHOTT Optical Glass Data Sheet. [Online]. http://www.us.schott.com/english/index.html

[20] J. Homola, "Surface Plasmon Resonance Sensors for Detection of Chemical and Biological Species," Chem. Rev., vol. 108, no. 2, pp. 462-493, 2008.

[21] X. Caide and S.-F. Sui, "Characterization of surface plasmon resonance biosensor," Sens. Actuators, B, vol. 66, no. 1, pp. 174-177, 2000.

[22] Y. Xinglong, W. Dingxin, and Y. Zibo, "Simulation and analysis of surface plasmon resonance biosensor based on phase detection," Sens. Actuators, B, vol. 91, no. 1-3, pp. 285-290, 2003.

[23] M. Vala, K. Chadt, M. Piliarik, and J. Homola, "High-performance compact SPR sensor for multi-analyte sensing," Sens. Actuators, B, vol. 148, no. 2, pp. 544-549, 2010.

[24] P. Berini, "Long-range surface plasmon polaritons," Advances in Optics and Photonics, vol. 1, no. 3, pp. 484-588, 2009.

[25] K. A. Willets and R. P. Van Duyne, "Localized Surface Plasmon Resonance Spectroscopy and Sensing," Annu. Rev. Phys. Chem., vol. 58, pp. 267-97, 2007.

[26] D. E. Mustafa et al., "Surface Plasmon Coupling Effect of Gold Nanoparticles with Different Shape and Size on Conventional Surface Plasmon Resonance Signal," Plasmonics, vol. 5, no. 3, pp. 221-231, 2010.

[27] J. Homola, Surface plasmon resonance based sensors, Springer series on chemical sensors and biosensors 4, Ed. Berlin: Springer, 2006.

[28] M. Piliarik and J. Homola, "Surface plasmon resonance (SPR) sensors: approaching their limits?," Opt. Express, vol. 17, no. 19, pp. 16505-16517, 2009.

[29] GE Healthcare Biacore. (2011) Biacore. [Online]. www.biacore.com

[30] M. Piliarik, H. Vaisocherová, and J. Homola, "Towards parallelized surface plasmon resonance sensor platform for sensitive detection of oligonucleotides," Sens. Actuators, B, vol. 21, no. 1, pp. 187-193, 2007. 
[31] G.G. Nenninger, M. Piliarik, and J. Homola, "Data analysis for optical sensors based on spectroscopy of surface plasmons," Meas. Sci. Technol., vol. 13, no. 12, pp. 2038-2046, 2002.

[32] R. Slavík and J. Homola, "Ultrahigh resolution long range surface plasmonbased sensor," Sens. Actuators, B, vol. 123, no. 1, pp. 10-12, 2007.

[33] P.I. Nikitin et al., "Surface plasmon resonance interferometry for micro-array biosensing," Sens. Actuators, A, vol. 85, no. 1, pp. 189-193, 2000.

[34] S.Y. Wu, H.P. Ho, W.C. Law, C. Lin, and S.K. Kong, "Highly sensitive differential phase-sensitive surface plasmon resonance biosensor based on the Mach-Zehnder configuration," Opt. Lett., vol. 29, no. 20, pp. 2378-2380, 2004.

[35] J. Dostálek, J. Homola, and M. Miler, "Rich information format surface plasmon resonance biosensor based on array of diffraction gratings," Sens. Actuators, B, vol. 107, no. 1, pp. 154-161, 2005.

[36] M.-H. Chiu, S.-F. Wang, and R.-S. Chang, "D-type fiber biosensor based on surface-plasmon resonance technology and heterodyne interferometry," Opt. Lett., vol. 30, no. 3, pp. 233-235, 2005.

[37] J. Dostálek et al., "Surface plasmon resonance biosensor based on integrated optical waveguide," Sens. Actuators, B, vol. 76, no. 1-3, pp. 8-12, 2001.

[38] F. Bardin, A. Bellemain, G. Roger, and M. Canva, "Surface plasmon resonance spectro-imaging sensor for biomolecular surface interaction characterization," Biosens. Bioelectron., vol. 24, no. 7, pp. 2100-2105, 2009.

[39] K. Johansen, "Surface plasmon resonance: instrumental resolution using photo diode arrays," Meas. Sci. Technol., vol. 11, no. 11, pp. 1630-1638, 2000 .

[40] H. Jiandong and Z. Xiangyang, "An improved centroid algorithm for a surface plasmon resonance bioanalyzer using microprocessors," in Symposium on Photonics and Optoelectronics, Wuhan, 2009, pp. 1 - 4.

[41] C. J. Alleyne, "Enhancing sensitivity for surface plasmon resonance biosensors using periodic structures and spectro-angular image analysis," Dept. Elect. Eng., McGill Univ., Montréal, PhD Thesis 2009.

[42] M. Daimon and A. Masumura, "Measurement of the refractive index of distilled water from the near-infrared region to the ultraviolet region," Appl. Opt., vol. 46, no. 18, pp. 3811-3820, 2007. 